On geometrical properties of preconditioners in IPMs for classes of block-angular problems

Jordi Castro

Dept. of Stat. and Oper. Res. Universitat Politècnica de Catalunya

Barcelona, Catalonia

jordi.castro@upc.edu
Stefano Nasini

Dept. of Marketing

IESEG School of Management

Lille, France

snasini@ieseg.fr

Research Report UPC-DEIO DR 2016-03

February 2016; updated November 2016 



\title{
ON GEOMETRICAL PROPERTIES OF PRECONDITIONERS IN IPMS FOR CLASSES OF BLOCK-ANGULAR PROBLEMS*
}

\author{
JORDI CASTRO $\dagger$ AND STEFANO NASINI $\ddagger$
}

\begin{abstract}
One of the most efficient interior-point methods for some classes of block-angular structured problems solves the normal equations by a combination of Cholesky factorizations and preconditioned conjugate gradient for, respectively, the block and linking constraints. In this work we show that the choice of a good preconditioner depends on geometrical properties of the constraints structure. In particular, it is seen that the principal angles between the subspaces generated by the diagonal blocks and the linking constraints can be used to estimate ex-ante the efficiency of the preconditioner. Numerical validation is provided with some generated optimization problems. An application to the solution of multicommodity network flow problems with nodal capacities and equal flows of up to 64 million variables and up to 7.9 million constraints is also presented.
\end{abstract}

Key words. interior-point methods, structured problems, preconditioned conjugate gradient, principal angles, large-scale optimization

AMS subject classifications. 90C06, 90C08, 90C51

1. Introduction. Many real-world optimization problems exhibit a primal block-angular structure, where decision variables and constraints can be grouped in different blocks which are dependent due to some linking constraints Applications of this class of problems can be found, for instance, in multicommodity telecommunications networks, statistical data protection, multistage control and planning, and complex networks.

Solution strategies to deal with this class of problems can be broadly classified into simplex-based methods [14, 23], decomposition methods [17, 1, 2, 27], approximation methods [5], and interiorpoint methods [10, 21]. One of the most efficient interior-point methods (IPMs) for some classes of block-angular problems solves normal equations by a combination of Cholesky factorizations for the block constraints and preconditioned conjugate gradient (PCG) iterations for the linking constraints $[10,11]$. The spectral radius of a certain matrix in the preconditioner, which is always in $[0,1)$ plays an important role in the efficiency of this approach. It was observed that for separable convex problems with nonzero Hessians this spectral radius is reduced, and the PCG becomes more efficient [13]. When the spectral radius approaches 1 , switching to a suitable preconditioner may be an efficient alternative [7]. It is worth noting that computing approximate Newton directions by PCG does not destroy the good convergence properties of IPMs, as shown in [20]. There is an extensive literature on the use of PCG within IPMs for other types of problems (e.g., $[3,4,9,18,25,28]$ to mention a few).

However, it is not yet clear why for some classes of block-angular problems the above approach may be very efficient (see, for instance, the results of $[13,12]$ ), while it may need a large number of PCG iterations in others. The spectral radius may be used to monitor the good or bad behaviour, but an ex-ante explanation has to be found in the structural information of the block-angular constraints matrix. The purpose of this paper is to provide such a explanation by considering geometrical properties of the primal block-angular matrix structure which, in addition, may be used to improve the preconditioning in some instances. It will be shown that the principal angles between the subspaces generated by the diagonal blocks and the ones generated by the linking constraints play an important role, explaining the efficiency of the approach and providing a proper choice of the preconditioner.

This paper is organized as follows. Section 2 outlines the specialized IPM for primal blockangular problems. Section 3 analyzes the quality of preconditioning techniques based on geometrical and spectral properties. This analysis is used to obtain complementary preconditioners to deal with orthogonality and collinearity of the subspaces generated by the diagonal blocks and the ones generated by the linking constraints. Section 4 provides a numerical validation of the analysis in previous section. This is based on both a simulation analysis of the effect of geometrical relations on PCG iterations - Subsection 4.1-and a real world application to multicommodity network flow problems with nodal capacities - Subsection 4.2-, where the analyzed geometrical properties can be fully exploited.

\footnotetext{
*This work has been supported by MINECO-FEDER grants MTM2012-31440 and MTM2015-65362-R.

${ }^{\dagger}$ Dept. of Statistics and Operations Research,Universitat Politècnica de Catalunya, Jordi Girona 1-3, 08034 Barcelona, Catalonia. jordi.castro@upc.edu

${ }^{\ddagger}$ Department of Marketing, IESEG School of Management (LEM-CNRS 9221), 3 rue de la Digue, 59000 Lille, France.s.nasini@ieseg.fr
} 
2. Outline of the IPM for primal block-angular problems. The primal block-angular formulation dealt with by the algorithm is

$$
\begin{aligned}
& \min \sum_{i=0}^{k}\left(c^{i}\right)^{\top} x^{i} \\
& \text { subject to }\left[\begin{array}{ccccc}
N_{1} & & & & \\
& N_{2} & & & \\
& & \ddots & & \\
& & & N_{k} & \\
L_{1} & L_{2} & \ldots & L_{k} & I
\end{array}\right]\left[\begin{array}{c}
x^{1} \\
x^{2} \\
\vdots \\
x^{k} \\
x^{0}
\end{array}\right]=\left[\begin{array}{c}
b^{1} \\
b^{2} \\
\vdots \\
b^{k} \\
b^{0}
\end{array}\right] \\
& 0 \leq x^{i} \leq u^{i} \quad i=0, \ldots, k \text {. }
\end{aligned}
$$

Matrices $N_{i} \in \mathbb{R}^{m_{i} \times n_{i}}$ and $L_{i} \in \mathbb{R}^{l \times n_{i}}, i=1, \ldots, k$, respectively define the block-diagonal and linking constraints, $k$ being the number of blocks. Vectors $x^{i} \in \mathbb{R}^{n_{i}}, i=1, \ldots, k$, are the variables for each block. $x^{0} \in \mathbb{R}^{l}$ are the slacks of the linking constraints. $b^{i} \in \mathbb{R}^{m_{i}}, i=1, \ldots, k$, is the right-hand side vector for each block of constraints, whereas $b^{0} \in \mathbb{R}^{l}$ is for the linking constraints. The upper bounds for each group of variables are defined by $u^{i} \in \mathbb{R}^{n_{i}}, i=0, \ldots, k$. Problems with equality linking constraints can be formulated by setting $u^{0}=0$. The total number of constraints and variables of (2.1) is thus, respectively, $\widetilde{m}=l+\sum_{i=1}^{k} m_{i}$ and $\widetilde{n}=l+\sum_{i=1}^{k} n_{i}$. Formulation (2.1) is a very general model which accommodates to several block-angular problems.

In this work we consider the specialized IPM of $[10,11]$. Briefly, this approach requires the solution at each interior-point iteration of the normal equations system $A \Theta A^{\top} \Delta y=g$, where $A \in \mathbb{R}^{\tilde{m} \times \widetilde{n}}$ is the constraints matrix of $(2.1 \mathrm{~b}) ; \Theta=\left(W(U-X)^{-1}+Z X^{-1}\right)^{-1} \in \mathbb{R}^{\tilde{n}}$ is a diagonal matrix computed from the values $(x, w, z) \in \mathbb{R}^{3 \widetilde{n}}$ of the current primal-dual point- $w$ and $z$ being the Lagrange multipliers associated to, respectively, upper and lower bounds; $\Delta y \in \mathbb{R}^{\widetilde{m}}$ is the direction of movement for the Lagrange multipliers of equality constraints $y$; and $g \in \mathbb{R}^{\tilde{m}}$ is some right-hand side. A derivation of the normal equations can be found in [29].

Exploiting the block structure of $A$ and $\Theta$ we have

$$
\begin{aligned}
& A \Theta A^{\top} \Delta y=\left[\begin{array}{ccc|c}
N_{1} \Theta_{1} N_{1}^{\top} & & & N_{1} \Theta_{1} L_{1}^{\top} \\
& \ddots & & \vdots \\
& & N_{k} \Theta_{k} N_{k}^{\top} & N_{k} \Theta_{k} L_{k}^{\top} \\
& & \\
\hline L_{1} \Theta_{1} N_{1}^{\top} & \ldots & L_{k} \Theta_{k} N_{k}^{\top} & \Theta_{0}+\sum_{i=1}^{k} L_{i} \Theta_{i} L_{i}^{\top}
\end{array}\right] \Delta y \\
& =\left[\begin{array}{cc}
B & C \\
C^{\top} & D
\end{array}\right]\left[\begin{array}{l}
\Delta y_{1} \\
\Delta y_{2}
\end{array}\right]=\left[\begin{array}{l}
g_{1} \\
g_{2}
\end{array}\right],
\end{aligned}
$$

where $\Delta y_{1}$ and $\Delta y_{2}$ are the components of $\Delta y$ associated to, respectively, block and linking constraints; $\Theta_{i}=\left(W_{i}\left(U_{i}-X_{i}\right)^{-1}+Z_{i} X_{i}^{-1}\right)^{-1}, i=0, \ldots, k$, are the blocks of $\Theta$; and $g=\left(g_{1}^{\top} g_{2}^{\top}\right)^{\top}$ is a proper partition of the right-hand side $g$. By eliminating $\Delta y_{1}$ from the first group of equations of (2.2), we obtain

$$
\begin{aligned}
\left(D-C^{\top} B^{-1} C\right) \Delta y_{2} & =\left(g_{2}-C^{\top} B^{-1} g_{1}\right) \\
B \Delta y_{1} & =\left(g_{1}-C \Delta y_{2}\right) .
\end{aligned}
$$

The specialized IPM for this class of problems solves (2.3) by a combination of $k$ Cholesky factorizations, for the systems involving $B$ and PCG for (2.3a). Indeed, matrix $D-C^{\top} B^{-1} C \in \mathbb{R}^{l \times l}$ of (2.3a), whose dimension is the number of linking constraints, is symmetric and positive definite, since it is the Schur complement of the normal equations (2.2), which are symmetric and positive definite. System (2.3a) can thus be solved by PCG. A good preconditioner is instrumental. $D-C^{\top} B^{-1} C$ is a $P$-regular splitting, i.e., it is symmetric and positive definite, $D$ is nonsingular and $D+C^{\top} B^{-1} C$ is positive definite. Therefore the $P$-regular splitting theorem [26] guarantees that

$$
0<\rho\left(D^{-1}\left(C^{\top} B^{-1} C\right)\right)<1,
$$

where $\rho(\cdot)$ denotes the spectral radius of a matrix (i.e., the maximum absolute eigenvalue). This allows us to compute the inverse of $D-C^{\top} B^{-1} C$ as the following infinite power series (see [10, Prop. 
4] for a proof).

$$
\left(D-C^{\top} B^{-1} C\right)^{-1}=\left(\sum_{i=0}^{\infty}\left(D^{-1}\left(C^{\top} B^{-1} C\right)\right)^{i}\right) D^{-1} .
$$

A preconditioner is thus obtained by truncating the infinite power series (2.5) at some term. The more the terms included, the better the preconditioner will be, at the expense of increasing the execution time of each PCG iteration. If we only include the first term, or the first and second terms, of the infinite power series (2.5) the resulting preconditioners will be, respectively, $D^{-1}$ or $\left(I+D^{-1}\left(C^{\top} B^{-1} C\right)\right) D^{-1}$. As shown in [12], in most test problems $D^{-1}$ was the best option for the tradeoff between being a good and an efficient preconditioner. Thus, in this work we will focus on the first term preconditioner $D^{-1}$. Although the expected performance for a general primal blockangular matrix is problem dependent, the effectiveness of the preconditioner obtained by truncating the infinite power series (2.5) is governed by the spectral radius $\rho\left(D^{-1}\left(C^{\top} B^{-1} C\right)\right)$-the farther from 1 , the better [13] — and the structure of $D$, as each PCG iteration requires the solution of a system with this matrix.

The spectral radius tends to approach 1 when the IPM is close to the optimal solution [13]. For this reason a hybrid preconditioner was introduced in [7]: the power series preconditioner (either $D^{-1}$ or $\left.\left(I+D^{-1}\left(C^{\top} B^{-1} C\right)\right) D^{-1}\right)$ is used in the initial IPM iterations, switching to the splitting preconditioner [25] when the former becomes inefficient. The above hybrid scheme is supported by the known good behaviour of the splitting preconditioner near the solution of the linear optimization problem. Briefly, given the normal equations matrix $A \Theta A^{\top}$, the splitting preconditioner is based on a partition of the columns of $A$ into basic and nonbasic columns, forming the nonsingular basis matrix $A_{B} \in \mathbb{R}^{\tilde{m} \times \widetilde{m}}$ and the nonbasic one $A_{N} \in \mathbb{R}^{\tilde{m} \times(\widetilde{n}-\widetilde{m})}$, respectively. Applying the same partition to $\Theta$, the normal equations matrix can be rewritten as

$$
A \Theta A^{\top}=A_{B} \Theta_{B} A_{B}^{\top}+A_{N} \Theta_{N} A_{N}^{\top} .
$$

The splitting preconditioner is defined as $\Theta_{B}^{-1 / 2} A_{B}^{-1}$. The symmetric application of this preconditioner to $A \Theta A^{\top}$ gives:

$$
\begin{aligned}
\left(\Theta_{B}^{-1 / 2} A_{B}^{-1}\right)\left(A \Theta A^{\top}\right)\left(\Theta_{B}^{-1 / 2} A_{B}^{-1}\right)^{\top} & =\Theta_{B}^{-1 / 2} A_{B}^{-1}\left(A_{B} \Theta_{B} A_{B}^{\top}+A_{N} \Theta_{N} A_{N}^{\top}\right) A_{B}^{-T} \Theta_{B}^{-1 / 2} \\
& =I+\left(\Theta_{B}^{-1 / 2} A_{B}^{-1} A_{N} \Theta_{N}^{1 / 2}\right)\left(\Theta_{B}^{-1 / 2} A_{B}^{-1} A_{N} \Theta_{N}^{1 / 2}\right)^{\top}
\end{aligned}
$$

Sufficiently close to an optimal solution, with a suitable choice of the columns of $A_{B}$, the diagonal entries of $\Theta_{B}^{-1}$ and $\Theta_{N}$ are very small and $\left(\Theta_{B}^{-1 / 2} A_{B}^{-1} A_{N} \Theta_{N}^{1 / 2}\right)$ approaches the zero matrix. Some strategies for identifying a suitable matrix $A_{B}$ are discussed in $[25,7]$.

This specialized IPM has been recently implemented in a software package called BlockIP [12], that will be used for the computational results of this paper.

3. Analysis of preconditioning techniques for block-angular problems. By the definition of $B, C$ and $D$ in $(2.2)$, we may write

$$
\begin{aligned}
C^{\top} B^{-1} C & =\sum_{i=1}^{k} L_{i} \Theta_{i} N_{i}^{\top}\left(N_{i} \Theta_{i} N_{i}^{\top}\right)^{-1} N_{i} \Theta_{i} L_{i}^{\top} \\
& =\sum_{i=1}^{k} L_{i} \Theta_{i}^{1 / 2} P_{i} \Theta_{i}^{1 / 2} L_{i}^{\top}
\end{aligned}
$$

where

$$
P_{i}=\Theta_{i}^{1 / 2} N_{i}^{\top}\left(N_{i} \Theta_{i} N_{i}^{\top}\right)^{-1} N_{i} \Theta_{i}^{1 / 2} \quad i=1, \ldots, k
$$

is the projection operator onto $\mathcal{R}\left(\Theta_{i}^{1 / 2} N_{i}^{\top}\right)$, the range or column space of $\Theta_{i}^{1 / 2} N_{i}^{\top}$. Let us consider the following two opposite cases:

1. If each column of $\Theta_{i}^{1 / 2} L_{i}^{\top}$ belongs to $\mathcal{R}\left(\Theta_{i}^{1 / 2} N_{i}^{\top}\right.$ ) for $i=1, \ldots, k$ (which is equivalent to saying that the rows of $L_{i} \Theta_{i}^{1 / 2}$ might be written as a linear combination of the rows of $N_{i} \Theta_{i}^{1 / 2}$ ) then $P_{i} \Theta_{i}^{1 / 2} L_{i}^{\top}=\Theta_{i}^{1 / 2} L_{i}^{\top}$, as $P_{i}$ is the identity operator of the subspace generated 


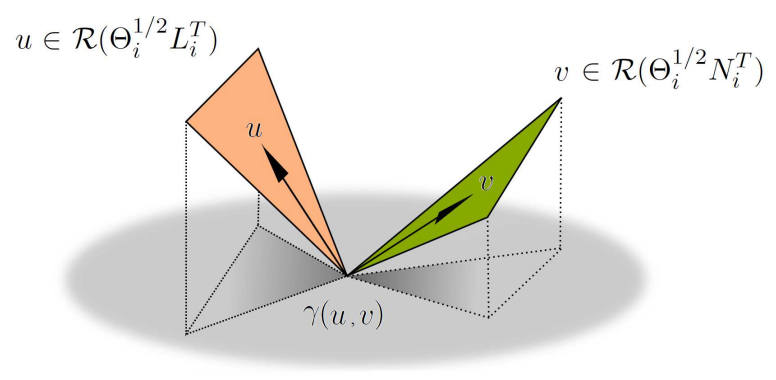

FIG. 1. Angles between subspaces.

by the columns of $\Theta_{i}^{1 / 2} N_{i}^{\top}$, and

$$
\begin{aligned}
D-C^{\top} B^{-1} C & =D-\sum_{i=1}^{k} L_{i} \Theta_{i}^{1 / 2} P_{i} \Theta_{i}^{1 / 2} L_{i}^{\top} \\
& =D-\sum_{i=1}^{k} L_{i} \Theta_{i} L_{i}^{\top}=\Theta_{0} .
\end{aligned}
$$

2. On the contrary, if each column of $\Theta_{i}^{1 / 2} L_{i}^{\top}$ belongs to $\mathcal{N}\left(N_{i} \Theta_{i}^{1 / 2}\right)$, the null space of $N_{i} \Theta_{i}^{1 / 2}$, for $i=1, \ldots, k$, then $P_{i} \Theta_{i}^{1 / 2} L_{i}^{\top}=0$ and

$$
D-C^{\top} B^{-1} C=D-\sum_{i=1}^{k} L_{i} \Theta_{i}^{1 / 2} P_{i} \Theta_{i}^{1 / 2} L_{i}^{\top}=D .
$$

Thus $\Theta_{0}^{-1}$ and $D^{-1}$ would be the inverse of $D-C^{\top} B^{-1} C$ (i.e., the exact preconditioners) when, for $i=1 \ldots k$, each column of $\Theta_{i}^{1 / 2} L_{i}^{\top}$ belongs to either $\mathcal{R}\left(\Theta_{i}^{1 / 2} N_{i}^{\top}\right)$ or $\mathcal{N}\left(N_{i} \Theta_{i}^{1 / 2}\right)$, respectively. Needless to say, these two extreme cases will rarely appear in a real problem; for instance, when $\Theta_{i}^{1 / 2} L_{i}^{\top}$ belongs to $\mathcal{R}\left(\Theta_{i}^{1 / 2} N_{i}^{\top}\right)$ the linking constraints are redundant and can be removed, obtaining a block-separable problem. However, as seen in next subsections, they allow to decide whether $D^{-1}$ or $\Theta_{0}^{-1}$ will be a good preconditioner for (2.3a), and which of them will theoretically behave better.

\subsection{Geometrical and spectral properties.}

According to previous discussion, the goodness of the approximation of $\Theta_{0}^{-1}$ and $D^{-1}$ to $(D-$ $\left.C^{\top} B^{-1} C\right)^{-1}$ might be measured by the principal angles between the range spaces of $\Theta_{i}^{1 / 2} N_{i}^{\top}$ and $\Theta_{i}^{1 / 2} L_{i}^{\top}$, for $i=1, \ldots, k$. The principal angles provide information about the relative position of two subspaces of an inner product space. Consider two subspaces $\mathcal{L}_{\Theta}$ and $\mathcal{N}_{\Theta}$ of $\mathbb{R}^{n}$, with $\operatorname{dim} \mathcal{L}_{\Theta}=l$, $\operatorname{dim} \mathcal{N}_{\Theta}=m$, and $q=\min \{l, m\}$. The principal angles between $\mathcal{L}_{\Theta}$ and $\mathcal{N}_{\Theta}$, denoted as $0 \leq \gamma_{1} \leq$ $\ldots \leq \gamma_{q} \leq \pi / 2$, are obtained by solving for $j=1, \ldots, q$ this sequence of optimization problems [6][19, Chapter 12]:

$$
\begin{aligned}
\cos \left(\gamma_{j}\right)=\max _{u, v} & u^{\top} v \\
\text { subject to } & \|u\|=1,\|v\|=1 \\
& u \in \mathcal{L}_{\Theta}, v \in \mathcal{N}_{\Theta} \\
& v^{\top} v_{k}=0, u^{\top} u_{k}=0, k=1 \ldots j-1,
\end{aligned}
$$

$u_{j} \in \mathbb{R}^{n}$ and $v_{j} \in \mathbb{R}^{n}$ being the optimal vectors. In other words, for $j=1$ the procedure finds the unit vectors $u_{1} \in \mathcal{L}_{\Theta}$ and $v_{1} \in \mathcal{N}_{\Theta}$ which minimize the angle between them-named $\gamma_{1}$. For $j>1$, the procedure considers the orthogonal complements of $\operatorname{span}\left\{u_{1}, \ldots, u_{j-1}\right\}$ in $\mathcal{L}_{\Theta}$ and of $\operatorname{span}\left\{v_{1}, \ldots, v_{j-1}\right\}$ in $\mathcal{N}_{\Theta}$, and computes a new pair of vectors $u_{j}, v_{j}$ in the above subspaces minimizing the angle $\gamma_{j}$. In our case, we have that $\mathcal{L}_{\Theta}=\mathcal{R}\left(\Theta^{1 / 2} L^{\top}\right)$ and $\mathcal{N}_{\Theta}=\mathcal{R}\left(\Theta^{1 / 2} N^{\top}\right)$, where $N=N_{i}$, $L=L_{i}$ and $\Theta=\Theta_{i}$ for some $i \in\{1, \ldots, k\}$. The vectors $\left\{u_{1}, \ldots, u_{q}\right\}$ and $\left\{v_{1}, \ldots, v_{q}\right\}$ are called principal vectors, associated to principal angles $\left\{\gamma_{1}, \ldots, \gamma_{q}\right\}$. The principal angles between subspaces can be graphically depicted as in Figure 1. If $q=m=l$, the distance between the equidimensional subspaces $\mathcal{L}_{\Theta}$ and $\mathcal{N}_{\Theta}$ is defined as $\sin \gamma_{q}=\sqrt{1-\cos ^{2} \gamma_{q}}$ [19, Chapter 2]. The Appendix shows a procedure to compute principal angles based on the singular value decomposition. This procedure is 
prohibitive for large optimization problems, but, as it will be shown later, it is not required in our optimization method.

As $\Theta_{i}, i=1, \ldots, k$, are different at each interior-point iteration, the principal angles between the subspaces $\mathcal{L}_{\Theta}$ and $\mathcal{N}_{\Theta}$ also change. Accordingly, the goodness of the approximation of $\Theta_{0}^{-1}$ and $D^{-1}$ to $\left(D-C^{\top} B^{-1} C\right)^{-1}$ dynamically changes along the interior-point iterations. Proposition 3.1 below provides a lower bound of the cosine of principal angles of $\mathcal{L}_{\Theta}$ and $\mathcal{N}_{\Theta}$ as a function of the principal angles of $\mathcal{L}=\mathcal{L}_{I}$ and $\mathcal{N}=\mathcal{N}_{I}$, that is, the subspaces $\mathcal{L}=\mathcal{R}\left(L_{i}^{\top}\right)$ and $\mathcal{N}=\mathcal{R}\left(N_{i}^{\top}\right)$ defined by matrices $L_{i}$ and $N_{i}$ without the scaling matrix $\Theta_{i}$.

Proposition 3.1. Consider the subspaces $\mathcal{L}=\mathcal{R}\left(L^{\top}\right) \subseteq \mathbb{R}^{n}$ and $\mathcal{N}=\mathcal{R}\left(N^{\top}\right) \subseteq \mathbb{R}^{n}$, and the associated subspaces $\mathcal{L}_{\Theta}=\mathcal{R}\left(\Theta^{1 / 2} L^{\top}\right) \subseteq \mathbb{R}^{n}$ and $\mathcal{N}_{\Theta}=\mathcal{R}\left(\Theta^{1 / 2} N^{\top}\right) \subseteq \mathbb{R}^{n}$ under the linear transformation $\Theta^{1 / 2}$, where $L=L_{i}, N=N_{i}$ and $\Theta=\Theta_{i}$ for some $i \in\{1, \ldots, k\}$, $\Theta$ thus being a positive diagonal matrix. Let $\gamma(u, v)$ be the first principal angle between $\mathcal{L}$ and $\mathcal{N}$, associated to principal vectors $u$ and $v$; and $\gamma_{\Theta}$ the first principal angle between $\mathcal{L}_{\Theta}$ and $\mathcal{N}_{\Theta}$. If $\mathcal{L}$ and $\mathcal{N}$ are not orthogonal, then

$$
\cos \left(\gamma_{\Theta}\right) \geq \max \left\{0, \frac{\Theta_{c}}{\Theta_{\max }} \cos (\gamma(u, v))\right\},
$$

where $\Theta_{\min }$ and $\Theta_{\max }$ are the smallest and largest diagonal components of $\Theta$, and $\Theta_{c} \in\left[-\Theta_{\max }, \Theta_{\max }\right]$. If in addition $u_{i} v_{i} \geq 0$ for all $i=1, \ldots, n$ then we have

$$
\cos \left(\gamma_{\Theta}\right) \geq \frac{\Theta_{\min }}{\Theta_{\max }} \cos (\gamma(u, v))>0 .
$$

Proof. Let $\gamma\left(\frac{\Theta^{1 / 2} u}{\left\|\Theta^{1 / 2} u\right\|}, \frac{\Theta^{1 / 2} v}{\left\|\Theta^{1 / 2} v\right\|}\right)$ be the angle between the unitary transformed vectors $\frac{\Theta^{1 / 2} u}{\left\|\Theta^{1 / 2} u\right\|} \in$ $\mathcal{L}_{\Theta}$ and $\frac{\Theta^{1 / 2} v}{\left\|\Theta^{1 / 2} v\right\|} \in \mathcal{N}_{\Theta}$. From the definition of principal angles as resulting from the maximization problem (3.5)-(3.8), and since vectors $\frac{\Theta^{1 / 2} u}{\left\|\Theta^{1 / 2} u\right\|}$ and $\frac{\Theta^{1 / 2} v}{\left\|\Theta^{1 / 2} v\right\|}$ are feasible for this problem, we have

$$
\cos \left(\gamma_{\Theta}\right) \geq \cos \left(\gamma\left(\frac{\Theta^{1 / 2} u}{\left\|\Theta^{1 / 2} u\right\|}, \frac{\Theta^{1 / 2} v}{\left\|\Theta^{1 / 2} v\right\|}\right)\right) .
$$

Since $u, v$ are principal vectors, by (3.6) $\|u\|=\|v\|=1$. Moreover, since principal angles are always in $[0, \pi / 2]$, and we are assuming $\mathcal{L}$ and $\mathcal{N}$ are not orthogonal, we have

$$
0<u^{\top} v=\|u\|\|v\| \cos (\gamma(u, v))=\cos (\gamma(u, v)) \leq 1 .
$$

By definition of inner product, by (3.12), using that for any square matrix $M$ and vector $w\|M w\| \leq$ $\|M\|\|w\|$, and that $\sqrt{\Theta_{\max }} \geq\left\|\Theta^{1 / 2}\right\|$,

$$
\begin{aligned}
\cos \left(\gamma\left(\frac{\Theta^{1 / 2} u}{\left\|\Theta^{1 / 2} u\right\|}, \frac{\Theta^{1 / 2} v}{\left\|\Theta^{1 / 2} v\right\|}\right)\right) & =\frac{u^{\top} \Theta v}{\left\|\Theta^{1 / 2} u\right\|\left\|\Theta^{1 / 2} v\right\|} \geq \frac{u^{\top} \Theta v}{\left\|\Theta^{1 / 2}\right\|^{2}\|u\|\|v\|} \geq \\
& \geq \frac{1}{\Theta_{\max }} \frac{u^{\top} \Theta v}{u^{\top} v} \cos (\gamma(u, v)) \geq \frac{u^{\top} \Theta v}{\Theta_{\max }} \cos (\gamma(u, v)) .
\end{aligned}
$$

Defining the set $\mathcal{P}=\left\{i \in\{1, \ldots, n\}: u_{i} v_{i} \geq 0\right\}$, since $\|u\|=\|v\|=1$ and $u^{\top} v \leq 1$, we have that

$$
0 \leq \sum_{i \in \mathcal{P}} u_{i} v_{i} \leq 1 \quad \text { and } \quad-1 \leq \sum_{i \notin \mathcal{P}} u_{i} v_{i} \leq 0
$$

Then, by (3.14),

$$
u^{\top} \Theta v=\sum_{i \in \mathcal{P}} \Theta_{i} u_{i} v_{i}+\sum_{i \notin \mathcal{P}} \Theta_{i} u_{i} v_{i} \geq \Theta_{\max } \sum_{i \notin \mathcal{P}} u_{i} v_{i} \geq-\Theta_{\max }
$$

and similarly,

$$
u^{\top} \Theta v=\sum_{i \in \mathcal{P}} \Theta_{i} u_{i} v_{i}+\sum_{i \notin \mathcal{P}} \Theta_{i} u_{i} v_{i} \leq \Theta_{\max } \sum_{i \in \mathcal{P}} u_{i} v_{i} \leq \Theta_{\max }
$$

Then, there exists some (unkwon) $\Theta_{c} \in\left[-\Theta_{\max }, \Theta_{\max }\right]$ such that

$$
\Theta_{c}=u^{\top} \Theta v \text {. }
$$


Applying (3.15) in (3.13), and since, by definition, $\cos \left(\gamma_{\Theta}\right) \geq 0$, inequality (3.9) is proved.

If $u_{i} v_{i} \geq 0$ for all $i=1, \ldots, n$, we have

$$
\frac{u^{\top} \Theta v}{u^{\top} v} \geq \frac{\Theta_{\min } u^{\top} v}{u^{\top} v}=\Theta_{\min } .
$$

Applying (3.16) to the penultimate term of (3.13) we get (3.10).

An important consequence of Proposition 3.1 is that we have no information about the principal angles in the final iterations of the IPM, as $\Theta_{\min } / \Theta_{\max }$ can approach 0 and $\Theta_{c} / \Theta_{\max }$ can be any number in $[-1,1]$ when the iterative process gets close to the optimal solution. In the worst case, it may even happen that almost-collinear subspaces $\mathcal{L}$ and $\mathcal{N}$ become almost-orthogonal for some $\Theta$.

It is worth noting that the two extreme cases have opposite behaviour:

- If $\mathcal{L}$ and $\mathcal{N}$ are orthogonal (that is, their principal angles are $\pi / 2$ ), then, for a diagonal $\Theta$ matrix such that some component $\Theta_{i, i}$ is very large, and for other components $j \neq i \Theta_{j j} \approx 0$, the principal angles of $\mathcal{L}_{\Theta}$ and $\mathcal{N}_{\Theta}$ will become close to 0 . This is a limit case of (3.10). In such a situation, $D^{-1}$ would be the best preconditioner for the first interior-point iterations, while $\Theta_{0}^{-1}$ should be used for the last ones. However, we empirically observed that, for the instances considered in this work, large principal angles in the first interior-point iterations may not tend to 0 as the algorithm approaches the solution. This will be illustrated in below Subsection 3.2 .

- If $\mathcal{L} \subseteq \mathcal{N}$, then the principal angles are 0. This means that, for some matrix $Y \in \mathbb{R}^{m \times l}$, $L^{\top}=N^{\top} Y$, thus $\left(\Theta^{1 / 2} L^{\top}\right)=\left(\Theta^{1 / 2} N^{\top}\right) Y$ and the principal angles of $\mathcal{L}_{\Theta}$ and $\mathcal{N}_{\Theta}$ will also be 0 . Although this case is not of practical interest-since if $\mathcal{L} \subseteq \mathcal{N}$ then the linking constraints are redundant and can be removed - it can explain, as we observed, why small principal angles in the initial interior-point iterations remain small near the optimal solution.

As shown below, the goodness of the dynamical approximation of $\Theta_{0}^{-1}$ and $D^{-1}$ to $(D-$ $\left.C^{\top} B^{-1} C\right)^{-1}$ along the interior-point iterations is related to the changes in the spectral radius of matrix $D^{-1}\left(C^{\top} B^{-1} C\right)$ - which is always in [0,1) [10, Theorem 1]. Since $D^{-1}$ is the first term of the power series (2.5), the farther away from 1 is the spectral radius of $D^{-1}\left(C^{\top} B^{-1} C\right)$ the better is the quality of the approximation of the first few terms of (2.5). Although the particular behavior of the spectral radius value is problem dependent, in general, it comes closer to 1 as we approach the optimal solution, because of the ill-conditioning of the $\Theta$ matrix. Next result provides a clear relationship between the spectral radius of $D^{-1}\left(C^{\top} B^{-1} C\right)$ and the projection operators in the subspaces $\mathcal{L}_{\Theta}$ and $\mathcal{N}_{\Theta}$.

Proposition 3.2. Let $\lambda$ be an arbitrary eigenvalue of $D^{-1}\left(C^{\top} B^{-1} C\right)$. If each column of $\Theta_{i}^{1 / 2} L_{i}^{\top}$ belongs to $\mathcal{R}\left(\Theta_{i}^{1 / 2} N_{i}^{\top}\right)$ then

$$
\lambda=\frac{r^{\top}\left(\sum_{i=1}^{k} L_{i} \Theta_{i} L_{i}^{\top}\right) r}{r^{\top} D r}
$$

where $r$ is an eigenvector associated to $\lambda$. On the contrary, if each column of $\Theta_{i}^{1 / 2} L_{i}^{\top}$ belongs to $\mathcal{N}\left(N_{i} \Theta_{i}^{1 / 2}\right)$, the null space of $N_{i} \Theta_{i}^{1 / 2}$, then

$$
\lambda=0 \text {. }
$$

Proof. Eigenvalue $\lambda$ of $D^{-1}\left(C^{\top} B^{-1} C\right)$ satisfies $\left(C^{\top} B^{-1} C\right) r=\lambda D r$ for some eigenvector $r$. From the definition of $C, B, D$ in (2.2) and projection matrices $P_{i}$ in (3.2) we have

$$
\left(\sum_{i=1}^{k} L_{i} \Theta_{i} N_{i}^{\top}\left(N_{i} \Theta_{i} N_{i}^{\top}\right)^{-1} N_{i} \Theta_{i} L_{i}^{\top}\right) r=\lambda D r
$$

is equivalent to

$$
(1-\lambda) D r=\left(\Theta_{0}+\sum_{i=1}^{k} L_{i} \Theta_{i} L_{i}^{\top}\right) r-\left(\sum_{i=1}^{k} L_{i} \Theta_{i} N_{i}^{\top}\left(N_{i} \Theta_{i} N_{i}^{\top}\right)^{-1} N_{i} \Theta_{i} L_{i}^{\top}\right) r
$$

which implies

$$
(1-\lambda)=\frac{r^{\top} \Theta_{0} r+r^{\top}\left(\sum_{i=1}^{k} L_{i} \Theta_{i}^{1 / 2}\left(I-P_{i}\right) \Theta_{i}^{1 / 2} L_{i}^{\top}\right) r}{r^{\top} D r}
$$


From this expression and applying the definition of $W_{\mathcal{R}}$ and $W_{\mathcal{N}}$

$$
W_{\mathcal{N}}=\sum_{i=1}^{k} L_{i} \Theta_{i}^{1 / 2}\left(I-P_{i}\right) \Theta_{i}^{1 / 2} L_{i}^{\top} \quad \text { and } \quad W_{\mathcal{R}}=\sum_{i=1}^{k} L_{i} \Theta_{i}^{1 / 2} P_{i} \Theta_{i}^{1 / 2} L_{i}^{\top}
$$

we find the following identities

$$
\lambda=\frac{r^{\top}\left(\sum_{i=1}^{k} L_{i} \Theta_{i} L_{i}^{\top}\right) r-r^{\top} W_{\mathcal{N} r}}{r^{\top} D r}=\frac{r^{\top} W_{\mathcal{R}} r}{r^{\top} D r} .
$$

It turns out that $r^{\top} W_{\mathcal{N}} r=0$ when each column of $\Theta_{i}^{1 / 2} L_{i}^{\top}$ belongs to $\mathcal{R}\left(\Theta_{i}^{1 / 2} N_{i}^{\top}\right)$; and $r^{\top} W_{\mathcal{R}} r=0$ when each column of $\Theta_{i}^{1 / 2} L_{i}^{\top}$ belongs to $\mathcal{N}\left(N_{i} \Theta_{i}^{1 / 2}\right)$. The proof is complete.

Note that a clear lesson from Proposition 3.2 is that the spectral radius might be small even in the case of almost collinearity between the rows of $L_{i} \Theta_{i}^{1 / 2}$ and their projections into $\mathcal{R}\left(\Theta_{i}^{1 / 2} N_{i}^{\top}\right)$, when $r^{\top} \Theta_{0} r \gg r^{\top}\left(\sum_{i=1}^{k} L_{i} \Theta_{i} L_{i}^{\top}\right) r$. This means that, although $\Theta_{0}$ is in theory a better preconditioner in case of almost collinearity, in practice it can be even outperformed by $D$. This is consistent with some of the computational results of Subsections 4.1-4.2, and with Theorem 1 of [13], which stated that the spectral radius $\rho$ of $D^{-1}\left(C^{\top} B^{-1} C\right)$ is bounded by

$$
0 \leq \rho \leq \max _{j \in\{1, \ldots, l\}} \frac{\gamma_{j}}{\left(\frac{u_{j}}{v_{j}}\right)^{2} \Theta_{0 j}+\gamma_{j}}<1
$$

where $u$ is the eigenvector (or one of the eigenvectors) of $D^{-1}\left(C^{\top} B^{-1} C\right.$ ) for $\rho ; \gamma_{j}, j=1, \ldots, l$, and $V=\left[V_{1} \ldots V_{l}\right]$, are respectively the eigenvalues and matrix of columnwise eigenvectors of $\sum_{i=1}^{k} L_{i} \Theta_{i} L_{i}{ }^{\top}$; $v=V^{\top} u$ (and, abusing of notation, we assume that for $\left.v_{j}=0,\left(u_{j} / v_{j}\right)^{2}=+\infty\right)$. Clearly, from (3.22), the larger $\Theta_{0}$, the closer $\rho$ to 0 , which it is also concluded from (3.17).

3.2. Practical estimation of principal angles. The use of principal angles between subspaces in our context has two downsides:

- If the subspace spanned by linking constraints is $\mathbb{R}^{n_{i}}$, that is, if $\mathcal{R}\left(L_{i}^{\top}\right)=\mathbb{R}^{n_{i}}$, for $i=1, \ldots, k$, then $\mathcal{N}_{i} \subseteq \mathcal{L}_{i}$. In such case the principal angles are always zero, regardless of what $\mathcal{R}\left(N_{i}^{\top}\right)$ is. This happens, for instance, in multicommodity network flow problems [10], edge-colored network problems [15], and cutting planes approaches for facility location problems [16], where $L_{i}=I$, for $i=1, \ldots, k$. Our interest is on whether the linking constraints belong to $\mathcal{R}\left(N_{i}^{\top}\right)$, equivalent to saying that the rows of $L_{i}$ may be written as a linear combination of the rows of $N_{i}$, whereas the principal angles between $\mathcal{R}\left(L_{i}^{\top}\right)$ and $\mathcal{R}\left(N_{i}^{\top}\right)$ might be seen as a measure of mutual dependency, in the sense that they go to zero even in the opposite case when the rows of $N_{i}$ are linear combinations of the ones of $L_{i}$. The latter condition does not imply any favorable argument for $\Theta_{0}$ as a preconditioner.

- As already stated in previous subsection, the computation of principal angles between $\mathcal{L}_{\Theta}$ and $\mathcal{N}_{\Theta}$ using the algorithm of Figure 7 in the Appendix can be totally prohibitive to be included as part of the optimization procedure.

To circumvent the above drawbacks, two sensible and more practical approaches will be described below. Both are based on the computation of principal angles between a subspace $\mathcal{L}_{\Theta_{i}}=\mathcal{R}\left(\Theta_{i}^{1 / 2} L^{\top}\right)$ of dimension 1 (that is, $L \in \mathbb{R}^{1 \times n_{i}}$ ) and $\mathcal{N}_{\Theta_{i}}=\mathcal{R}\left(\Theta_{i}^{1 / 2} N_{i}^{\top}\right)$ (where $N_{i} \in \mathbb{R}^{m_{i} \times n_{i}}$ ). In this case, the problem (3.5)-(3.8) is highly simplified. First, $q=\min \left\{1, m_{i}\right\}=1$, and then we do not have constraints (3.8) (i.e., there is only a principal angle to compute). Second, constraints (3.6) and (3.7) impose

$$
u=\Theta_{i}^{1 / 2} L^{\top} \alpha, \text { for some } \alpha \in \mathbb{R} \text {, and } 1=u^{\top} u=\alpha^{2} L \Theta_{i} L^{\top}, \text { such that } \alpha=\frac{1}{\sqrt{L \Theta_{i} L^{\top}}} .
$$

Similarly, we have

$$
v=\Theta_{i}^{1 / 2} N_{i}^{\top} x \text {, for some } x \in \mathbb{R}^{m_{i}} \text {, and } v^{\top} v=x^{\top} N_{i} N_{i}^{\top} x=1 .
$$

Then, using (3.23) and (3.24) the optimization problem (3.5)-(3.8) reduces to

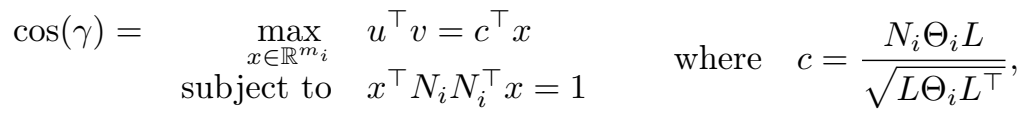


whose solution can be directly computed, considering a Lagrange multiplier $\nu \in \mathbb{R}$ for the constraint, as

$$
x^{*}=\left(N_{i} \Theta_{i} N_{i}^{\top}\right)^{-1} \frac{c}{2 \nu^{*}} \quad \text { where } \quad \nu^{*}=\frac{1}{2} \sqrt{c^{\top}\left(N_{i} \Theta_{i} N_{i}^{\top}\right)^{-1} c} .
$$

Since for the principal angle we only need the optimal value of the objective function $\cos (\gamma)=c^{\top} x^{*}$, computations can be simplified by noting that

$$
\cos (\gamma)=c^{\top} x^{*}=\frac{c^{\top}\left(N_{i} \Theta_{i} N_{i}^{\top}\right)^{-1} c}{\sqrt{c^{\top}\left(N_{i} \Theta_{i} N_{i}^{\top}\right)^{-1} c}}=\sqrt{c^{\top}\left(N_{i} \Theta_{i} N_{i}^{\top}\right)^{-1} c} .
$$

The cost of solving (3.26) is equivalent to the solution of a system of equations with one of the blocks defining matrix $B$ in (2.2), whose factorization is already available at each interior-point iteration.

The two practical approaches considered for computing principal angles, which rely on (3.26), are as follows:

- For each block $i, i=1, \ldots, k$, and linking constraint $j, j=1, \ldots, l$, we solve (3.26) for $L=L_{i, j}$, that is, we compute the principal angle-denoted as $\gamma_{i, j}$-between $N_{i}$ and the $j$-th row of $L_{i}$. Then the reported principal angle is the average of principal angles:

$$
\gamma=\frac{\sum_{i=1}^{k} \sum_{j=1}^{l} \gamma_{i, j}}{k l} .
$$

The inconvenience of this approach is that it solves $k l$ problems (3.26). A good feature of this method is that it averages angles between real linking constraints and block matrices.

- To reduce the number of problems (3.26) to be solved, in this second method we compute, for each block $i, i=1, \ldots, k$, the principal angle between $N_{i}$ and the average of the rows of $L_{i}$, that is we solve (3.26) for $L=\bar{L}_{i}=\left(\sum_{j=1}^{l} L_{i, j}\right) / l$. If we denote this angle as $\bar{\gamma}_{i}$, then the final angle provided is

$$
\gamma=\frac{\sum_{i=1}^{k} \bar{\gamma}_{i}}{k}
$$

This approach only requires solving $k$ problems (3.26). However, it provides some sort of approximation of the principal angle, since it does not use the rows of $L_{i}$, but a convex combination of them. This can be justified by noting that, if the principal angle between every row $L_{i, j}, j=1, \ldots, l$, and $N_{i}$ is 0 (that is, $L_{i, j}$ is linearly dependent with the rows of $N_{i}$ ) then the principal angle between a convex combination of rows of $L_{i}$ and $N_{i}$ will also be 0 .

The above two approaches have been implemented in the optimization package used for the computational experiments. Figure 2 shows the estimation of principal angles obtained with either (3.27) or (3.28) during the interior-point iterations of four of the instances of Tables $3-6$ of Subsection 4.2. We observe that, for the first interior-point iterations, both methods provide similar values. However, when we approach the optimal solution, angles computed with (3.28) tend to go to 0, while those obtained with (3.27) in general remain close to the original values. From Table 7 of Subsection 4.2 - which provides the true angles for these instances, computed using the costly algorithm in Figure 7 of the Appendix - we see that angles computed with (3.27) are usually better estimations. Unfortunately, in the huge instances of Subsection 4.2, method (3.27) was prohibitive (it resulted in very high CPU times), so we had to (successfully) apply method (3.28). The estimations with (3.27) in Figure 2 also confirm, as discussed in previous subsection, that although the scaling matrices $\Theta_{i}$ may change the principal angles at each interior-point iteration, in general when the angles are large at the first iterations, they remain large near the optimal solution.

There are other possible methods to reduce the computational cost of (3.27), such as, for instance, to consider just a sample or a representative subset of rows of $L_{i}$. However, we did not try these other options because, at least for the instances tested in this work, the results obtained with (3.28) were satisfactory.

According to the above discussion, if we want to predict from the beginning which preconditioner will behave better based on principal angles, we can use either (3.27) or (3.28), since both methods provide similar estimations at the first interior-point iterations. It is worth to mention that in this case (3.26) is solved before the IPM has started, and then $\Theta_{i}=I$ has to be used. Moreover, since $\Theta_{i}$ are the same for all $i=1, \ldots, k$, if matrices $N_{i}$ and $L_{i}$ are also the same for all $i$, then the number 


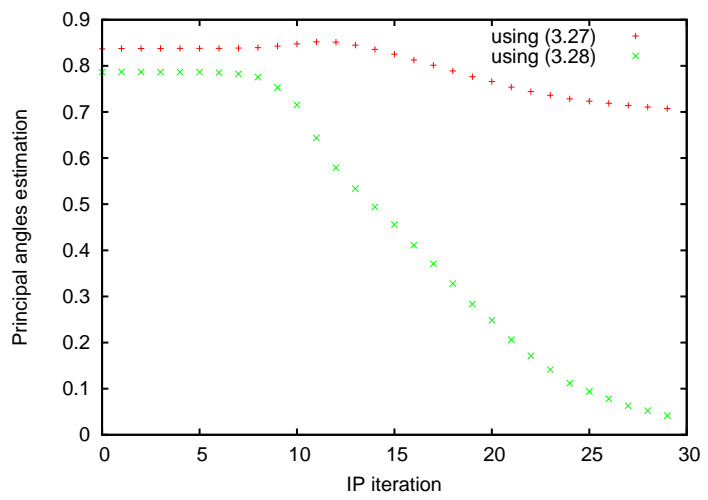

(a)

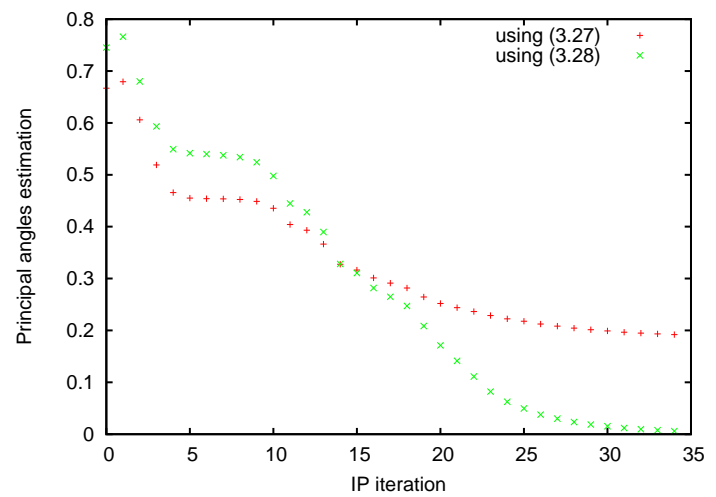

(c)

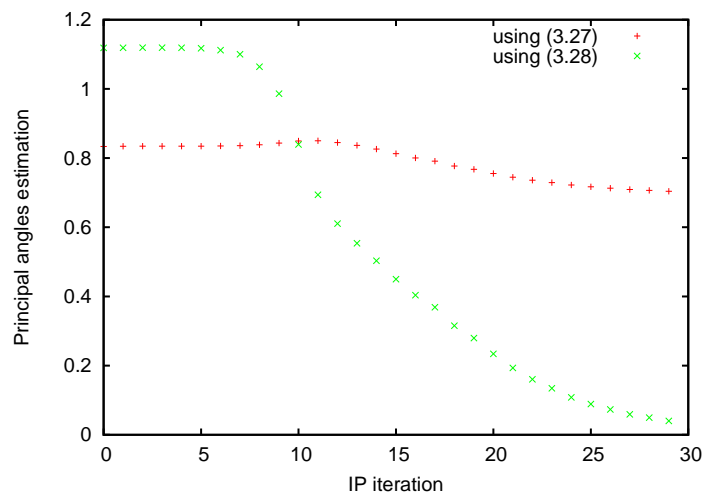

(b)

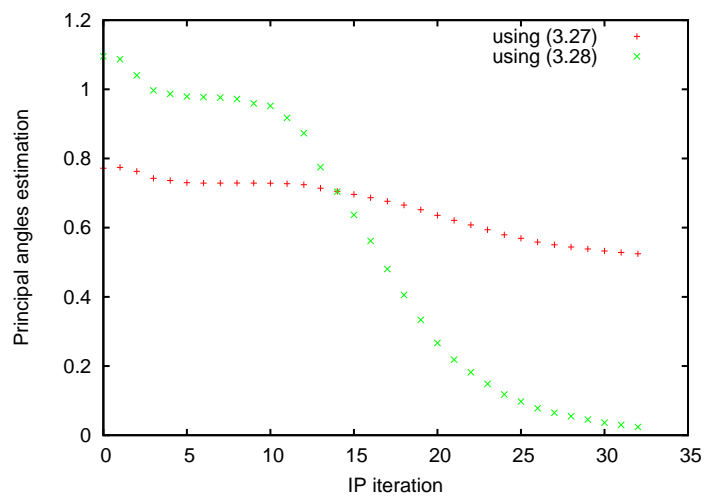

(d)

FIG. 2. Estimation of principal angles (in vertical axes) computed with either (3.27) or (3.28) along the interiorpoint iterations (in horizontal axes), for four particular instances. Plots (a) and (b) correspond to the first instances of, respectively, Tables 3 and 4 of Subsection 4.2. Plots (c) and (d) were obtained by adding extra constraints to the first instances of Tables 5 and 6 of Subsection 4.2.

of problems (3.26) to be solved drops to $l$ and 1, for, respectively, (3.27) and (3.28). We considered the following rule for automatic selection of the preconditioner:

$$
\begin{aligned}
& \text { if } \gamma \geq \tau_{\gamma} \text { then use } D^{-1}, \\
& \text { if } \gamma<\tau_{\gamma} \text { then use } \Theta_{0}^{-1} \text {, }
\end{aligned}
$$

where $\gamma$ has been previously computed with either (3.27) or (3.28) using $\Theta_{i}=I$, and $\tau_{\gamma}$ is a threshold parameter provided by the user. In the computational experiments of Subsection 4.2 we used $\tau_{\gamma}=$ $0.95 \pi / 4$, i.e., if the angle is closer to 0 than to $\pi / 2$, we choose $\Theta_{0}^{-1}$, otherwise $D^{-1}$ is selected; the 0.95 coefficient guarantees that, in case of tie, $D^{-1}$ will be used, since, according to (2.5), it is the first term of the exact preconditioner.

Switching between preconditioners at some interior-point iteration will only be worth if principal angles significantly change with $\Theta_{i}$. However, according to the above discussion, when the initial principal angles are close enough to $0, \Theta_{0}^{-1}$ will be in general the best preconditioner, not only for the first, but for all the interior-point iterations. Otherwise, $D^{-1}$ will be in general preferred. In addition, the CPU time required for computing principal angles is not negligible for huge instances. Given all these considerations, our switching criteria only computed principal angles if strictly necessary, and it focused on other indicators such as (1) the number of PCG iterations performed with the current preconditioner in the last interior-point iteration; (2) and, if the current preconditioner is $D^{-1}$, the spectral radius $\rho$ (which can be easily computed using the procedure described in [7]). The implemented switching criteria, which is applied every $\tau_{t}$ interior-point iterations, is as follows:

$$
\begin{aligned}
& \text { if current preconditioner is } \Theta_{0}^{-1} \text { then } \\
& \text { if (PCG iterations } \left.>\tau_{P} \cdot l\right) \text { and }\left(\gamma \geq \tau_{\gamma}\right) \text { then switch to } D^{-1} \text {, } \\
& \text { else if current preconditioner is } D^{-1} \text { then } \\
& \text { if }\left(\mathrm{PCG} \text { iterations }>\tau_{P} \cdot l\right) \text { and }\left(\rho>\tau_{\rho}\right) \text { and }\left(\gamma<\tau_{\gamma}\right) \text { then switch to } \Theta_{0}^{-1} \text {, }
\end{aligned}
$$

where $\tau_{\rho}$ is a threshold value for the spectral radius, $\tau_{P}$ is a percentage of the number of linking constraints $l$ (the size of the system solved by PCG), and $\tau_{\gamma}$ was already used in (3.29). The values 
TABLE 1

CPU time of BlockIP using $D^{-1}$ and $\Theta_{0}^{-1}$ preconditioners. The instances have $l=100$ linking constraints and $k=100$ equal diagonal block matrices $N \in \mathbb{R}^{10 \times 50}$.

\begin{tabular}{cccccccccc}
\hline \multirow{2}{*}{$\alpha$} & $\bar{r}$ & \multicolumn{2}{c}{ CPU time } & & \multicolumn{2}{c}{ IPM iterations } & & \multicolumn{2}{c}{ average PCG iter. } \\
& & $\Theta_{0}^{-1}$ & $D^{-1}$ & & $\Theta_{0}^{-1}$ & $D^{-1}$ & & $\Theta_{0}^{-1}$ & $D^{-1}$ \\
\hline$\pi / 14$ & 2 & 0.99 & 1.47 & & 30 & 43 & & 2.84 & 5.87 \\
$\pi / 10$ & 4 & 1.27 & 1.54 & & 38 & 44 & & 4.94 & 6.29 \\
$\pi / 6$ & 6 & 1.30 & 1.58 & & 36 & 46 & & 9.00 & 6.05 \\
$\pi / 2$ & 8 & 1.29 & 1.27 & & 35 & 39 & & 8.91 & 5.05 \\
\hline
\end{tabular}

TABLE 2

CPU time of BlockIP using $D^{-1}$ and $\Theta_{0}^{-1}$ preconditioners. The instances have $l=200$ linking constraints and $k=1000$ equal diagonal block matrices $N \in \mathbb{R}^{20 \times 200}$.

\begin{tabular}{ccrrrrrrrr}
\hline \multirow{2}{*}{$\alpha$} & \multirow{r}{*}{} & \multicolumn{2}{c}{ CPU time } & & \multicolumn{2}{c}{ IPM iterations } & & \multicolumn{2}{c}{ average PCG iter. } \\
& & $\Theta_{0}^{-1}$ & $D^{-1}$ & & $\Theta_{0}^{-1}$ & $D^{-1}$ & & $\Theta_{0}^{-1}$ & $D^{-1}$ \\
\hline$\pi / 14$ & 2 & 191.52 & 547.21 & & 28 & 77 & & 1.85 & 7.08 \\
$\pi / 10$ & 4 & 512.61 & 479.12 & & 80 & 61 & & 6.40 & 12.05 \\
$\pi / 6$ & 6 & 496.39 & 574.33 & & 64 & 76 & & 17.50 & 11.75 \\
$\pi / 2$ & 8 & 565.52 & 497.19 & & 67 & 70 & & 26.29 & 11.85 \\
\hline
\end{tabular}

used in the computational results of Subsection 4.2 were $\tau_{t}=3, \tau_{P}=0.2$, and $\tau_{\rho}=0.95$. As it will be observed in the computational results this switching criteria is rarely applied (only in three out of 60 instances). Choosing the right preconditioner, either $D^{-1}$ or $\Theta_{0}^{-1}$, from the beginning turned out to be a more important factor in the performance of the IPM. We remark that this situation is quite different from other hybrid preconditioning approaches, such as, for instance, the one in [7]: in that approach the two different preconditioners are known in advance to behave better for, respectively, the first and last interior-point iterations, and thus (1) we know in advance which preconditioner to start with; and (2) the switching is usually performed.

4. Numerical validation. The numerical validation of the proposed preconditioning technique is based on two types of computational experiments: (1) assessing the effect of the described geometrical relations to the number of PCG iterations; (2) analyzing the global numerical performance of both preconditioning techniques, when applied to classes of multicommodity network flow problems. All the runs were carried out on a Fujitsu Primergy RX300 server with two $3.33 \mathrm{GHz}$ Intel Xeon X5680 CPUs (each CPU with 12 cores) and 144 GB of RAM, under a GNU/Linux operating system (Suse 11.4), without exploitation of multithreading capabilities.

4.1. Geometrical relations and PCG iterations. Our goal here is to generate synthetic problems such that we can control the level of collinearity between their linking and block constraints. To this end, consider two full rank matrices $N \in \mathbb{R}^{m \times n}, n>m$, and $Y \in \mathbb{R}^{l \times m}, n>l$, and let $L=Y N$. The rows of $L \in \mathbb{R}^{l \times n}$ are linear combinations of the rows of $N$ and the principal angles between the subspaces generated by $L^{\top}$ and $N^{\top}$ are zero. Each vector in $\mathcal{R}\left(L^{\top}\right)$ can be rotated an angle $\alpha$ around the $i$-th and $j$-th coordinate axes by pre-multiplying $L^{\top}$ by the $n \times n$ rotation matrix:

$$
R_{i j}(\alpha)=\left[\begin{array}{ccccccccc}
1 & \cdots & 0 & 0 & \cdots & 0 & 0 & \cdots & 0 \\
\vdots & \ddots & \vdots & 0 & \cdots & 0 & \vdots & \ddots & \vdots \\
0 & \cdots & \cos (\alpha) & 0 & \cdots & 0 & -\sin (\alpha) & \cdots & 0 \\
0 & \cdots & 0 & 1 & \cdots & 0 & 0 & \cdots & 0 \\
\vdots & \ddots & \vdots & \vdots & \cdots & \vdots & \vdots & \ddots & \vdots \\
0 & \cdots & 0 & 0 & \cdots & 1 & 0 & \cdots & 0 \\
0 & \cdots & \sin (\alpha) & 0 & \cdots & 0 & \cos (\alpha) & \cdots & 0 \\
\vdots & \ddots & \vdots & \vdots & \cdots & \vdots & \vdots & \ddots & \vdots \\
0 & \cdots & 0 & 0 & \cdots & 0 & 0 & \cdots & 1
\end{array}\right] .
$$

If we consider $\mathcal{H}=\{(i, j): 1 \leq i \leq n-1, i<j \leq n\}$, the set of all possible pairs of coordinate axes, the $n(n-1) / 2$ distinct $R_{i j}(\alpha)$ rotation matrices may be concatenated in some order to produce a new rotation matrix such as $\prod_{(i, j) \in \mathcal{S}} R_{i j}(\alpha)$, where $\mathcal{S} \subseteq \mathcal{H}$. (Rotations in three dimensions and higher do not commute, so that different orderings give different rotations.)

We are interested in showing the performance of the PCG method at each interior-point iteration when changing the geometrical relations between the diagonal block and the linking constraint matrices, in accordance with specified rotations. To do so we randomly draw $N_{i} \in \mathbb{R}^{m \times n}$ and 
small instances

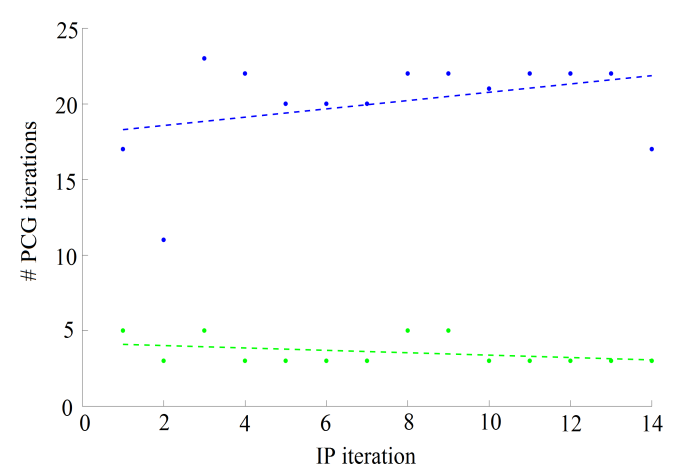

big instances

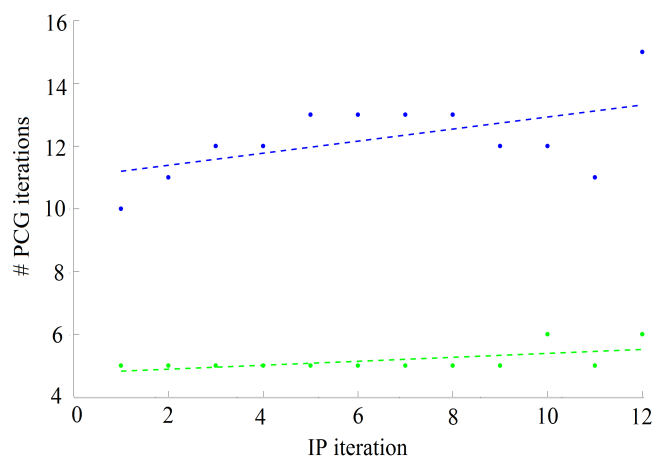

FIG. 3. PCG iterations along the IPM iterations for $\alpha=\pi / 16, \bar{r}=2$, with preconditioners $\Theta_{0}^{-1}$ and $D^{-1}$. The blue points (and associated dashed regression line) correspond to the PCG iterations based on $D^{-1}$ preconditioner, while the green points (and associated dashed regression line) correspond to the PCG iterations based on $\Theta_{0}^{-1}$ preconditioner.

small instances

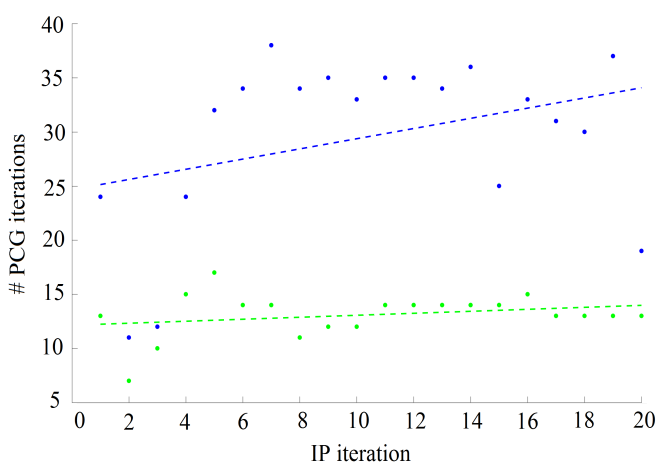

big instances

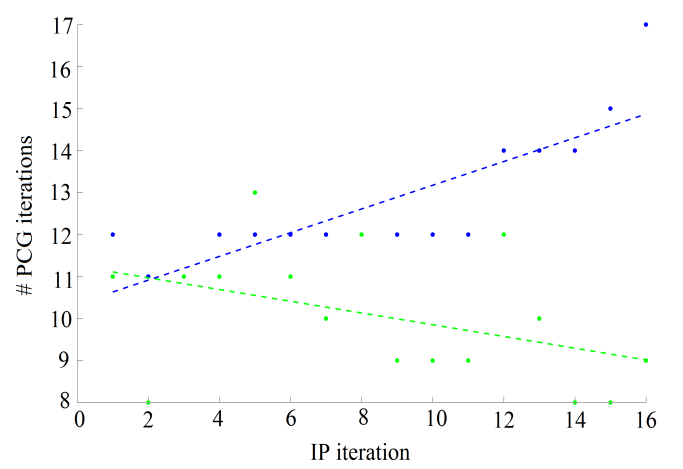

FIG. 4. PCG iterations along the IPM iterations for $\alpha=\pi / 12, \bar{r}=2$, with preconditioners $\Theta_{0}^{-1}$ and $D^{-1}$. The blue points (and associated dashed regression line) correspond to the PCG iterations based on $D^{-1}$ preconditioner, while the green points (and associated dashed regression line) correspond to the PCG iterations based on $\Theta_{0}^{-1}$ preconditioner.

$Y_{i} \in \mathbb{R}^{l \times m}, i=1, \ldots, k$, from a uniform probability distribution. Then $\mathcal{S} \subseteq \mathcal{H}$ is also randomly selected to compute the rotation matrix $\prod_{(i, j) \in \mathcal{S}} R_{i j}(\alpha)$. Finally, we obtain the linking constraint matrices $L_{i}=Y_{i} N_{i}\left(\prod_{(i, j) \in \mathcal{S}} R_{i j}(\alpha)\right)$, for $i=1, \ldots, k$. This procedure relies on the number $\bar{r}=|\mathcal{S}|$ of concatenated rotation matrices and the angle of rotation $\alpha$ to evaluate the associated changes in the PCG iterations using $\Theta_{0}^{-1}$ and $D^{-1}$ as preconditioners. It is worth to remark that $\alpha$ are not the principal angles between linking constraints matrices $L_{i}$ and block constraints matrices $N_{i}$, but it is expected that small $\alpha$ values (that is, small rotations) will result in small principal angles.

The BlockIP package [12] implementing the specialized interior-point method has been extended with the $\Theta_{0}^{-1}$ preconditioner introduced in this work. The main features of BlockIP are: (1) it implements a long-step infeasible IPM, including both Newton and predictor-corrector directions [29]; (2) it uses the approach described in Section 2-which relies on Cholesky factorizations and PCG - for the solution of the normal equations [10, 11, 13]; (3) Cholesky systems are exactly solved; (4) PCG systems are stopped when the error (computed as $1-\cos (Q x, d)$, for some system $Q x=d$ ) is below some tolerance; (5) in the computational results of this paper the initial PCG tolerance was set to $\epsilon^{0}=10^{-4}$, and it was updated at each interior-point iteration $t$ as $\epsilon^{t+1}=\min \left\{0.95 \cdot \epsilon^{t}, 10^{-8}\right\}$. More details about the code - which is available for research purposes from http://www-eio.upc.es/ jcastro/BlockIP.html—can be found in [12].

Tables 1 and 2 show the computational results obtained with BlockIP using $D^{-1}$ and $\Theta_{0}^{-1}$ preconditioners. Instances of Table 1 are "small", with $l=100$ linking constraints, and $k=100$ equal diagonal block matrices $N \in \mathbb{R}^{10 \times 50}$. For the "big" instances of Table 2 these dimensions are $l=200$, $k=1000$, and $N \in \mathbb{R}^{20 \times 200}$. The first columns of these tables show the angle $\alpha$ of each rotation, 
small instances

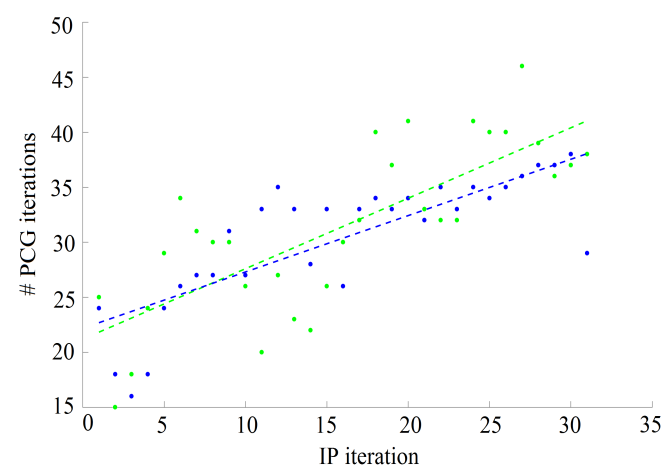

big instances

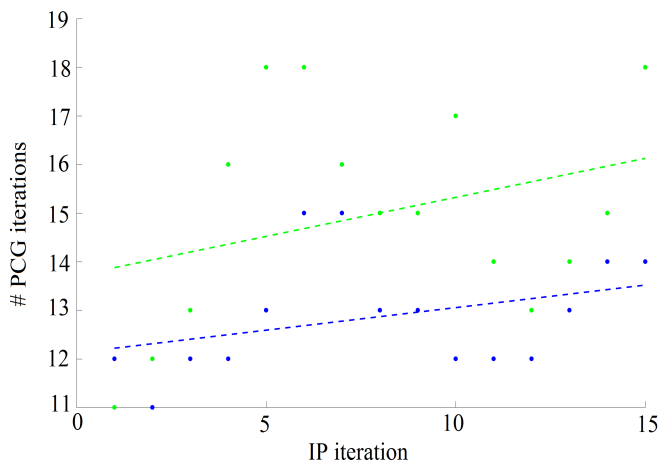

FIG. 5. PCG iterations along the IPM iterations for $\alpha=\pi / 8, \bar{r}=2$, with preconditioners $\Theta_{0}^{-1}$ and $D^{-1}$. The blue points (and associated dashed regression line) correspond to the PCG iterations based on $D^{-1}$ preconditioner, while the green points (and associated dashed regression line) correspond to the PCG iterations based on $\Theta_{0}^{-1}$ preconditioner.

small instances

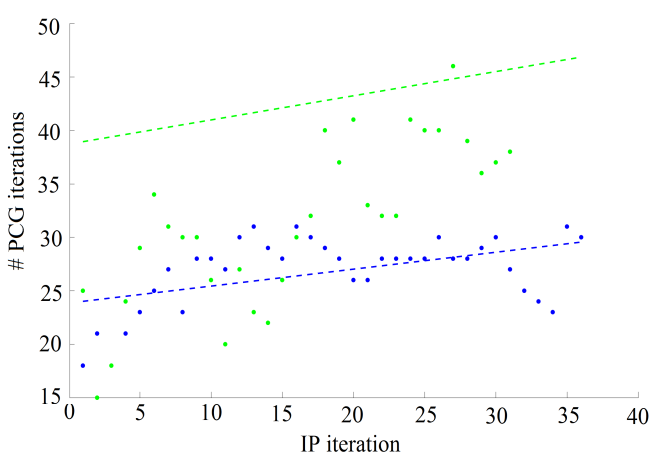

big instances

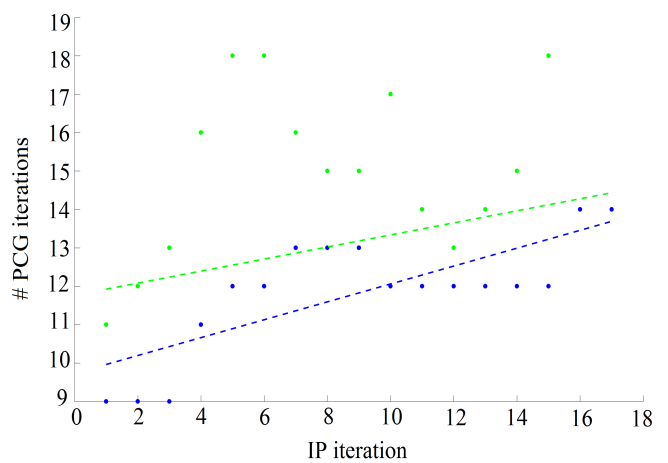

FIG. 6. PCG iterations along the IPM iterations for $\alpha=\pi / 4, \bar{r}=2$, with preconditioners $\Theta_{0}^{-1}$ and $D^{-1}$. The blue points (and associated dashed regression line) correspond to the PCG iterations based on $D^{-1}$ preconditioner, while the green points (and associated dashed regression line) correspond to the PCG iterations based on $\Theta_{0}^{-1}$ preconditioner.

whereas the second columns report the number $\bar{r}$ of rotation matrices considered. The remaining columns give the CPU time, number of IPM iterations, and average number of PCG iterations per IPM iteration, for both preconditioners.

It is observed that the performance of the specialized IPM strongly relies on $\alpha$ for both the small and big instances. In particular, for $\Theta_{0}^{-1}$, the smaller $\alpha$, the better the preconditioner; and the average number of PCG iterations increases with $\alpha$. On the other hand, $D^{-1}$ is the best precondtioner when $\alpha=\pi / 2$. This numerical evidence supports the previous discussion on the effect of the geometrical relations between the diagonal blocks and the linking constraint matrices on the quality of the preconditioner. In addition, the same conclusion will be provided by the numerical results of next Subsection 4.2 using a nontrivial-i.e., non-synthetic-problem.

The plots of Figures 3, 4, 5 and 6 illustrate the evolution of the number of PCG iterations (vertical axis) at each IPM iteration (horizontal axis) for both preconditioners. Small and large instances of the same dimensions as in Tables 1 and 2 have been used, but with different angles and always with $\bar{r}=2$ rotations matrices. The blue and green dots are related to $D^{-1}$ and $\Theta_{0}^{-1}$ respectively. The corresponding regression lines have been fitted to the empirical number of PCG iterations. It is observed that for small angles Figures 3 and 4), as expected by the theory, the preconditioner $D^{-1}$ usually requires more PCG iterations than $\Theta_{0}^{-1}$; this difference increases as we approach the optimal solution, for the small instances. However, for the "larger" angles of Figures 5 and 6 , again in accordance with theory, preconditioner $D^{-1}$ becomes more efficient than $\Theta_{0}^{-1}$ after certain IPM iteration. The message should then be that, for problems with small angles, $\Theta_{0}^{-1}$ should be preferred in general to $D^{-1}$. It is worth noting that a side benefit of $\Theta_{0}^{-1}$ is that it is always a diagonal preconditioner, independently of the problem, unlike $D^{-1}$, which may require a Cholesky 
TABLE 3

$C P U$ time and iterations (within parenthesis) of BlockIP (using $D^{-1}$ and $\Theta_{0}^{-1}$ preconditioners) and CPLEX available LP methods (Primal Simplex, Dual Simplex, Barrier Method) when solving MNFPNCs. Only $20 \%$ of nodes are constrained to have an outflow capacity, i.e., $l=0.2 n$.

\begin{tabular}{|c|c|c|c|c|c|c|c|c|}
\hline \multirow{2}{*}{$n$} & \multirow{2}{*}{$k$} & \multirow{2}{*}{ n. var. } & \multirow{2}{*}{ n. con. } & \multicolumn{3}{|c|}{ CPLEX } & \multicolumn{2}{|c|}{ BlockIP } \\
\hline & & & & Primal Simplex & Dual Simplex & Barrier & $\Theta_{0}^{-1}$ & $D^{-1}$ \\
\hline 150 & 200 & 880030 & 29830 & $57.5(997332)$ & $5.5(85732)$ & $5.8(13)$ & $5.7(29)$ & $5.6(29)$ \\
\hline 150 & 400 & 1803230 & 59630 & $242.2(428032)$ & $14.0(163391)$ & $12.9(14)$ & $12.2(30)$ & $12.1(30)$ \\
\hline 150 & 600 & 2601030 & 89430 & $362.2(444258)$ & $25.9(249271)$ & $19.7(15)$ & $19.6(31)$ & $18.8(31)$ \\
\hline 150 & 800 & 3850430 & 119230 & $590.5(575356)$ & $38.0(329632)$ & $30.5(16)$ & $24.1(29)$ & $26.8(32)$ \\
\hline 300 & 200 & 3587460 & 59860 & $459.3(4676110)$ & $27.5(203873)$ & $29.8(15)$ & $30.3(30)$ & $32.2(32)$ \\
\hline 300 & 400 & 7022460 & 119660 & $>3600(8306650)$ & $69.5(415156)$ & $57.2(15)$ & $57.4(30)$ & $59.1(30)$ \\
\hline 300 & 600 & 11245260 & 179460 & $>3600(8453150)$ & $112.7(571964)$ & $102.7(17)$ & $99.0(31)$ & $94.9(30)$ \\
\hline 300 & 800 & 14862460 & 239260 & $>3600(9071240)$ & $178.5(784104)$ & $140.3(18)$ & $123.1(30)$ & $133.8(31)$ \\
\hline
\end{tabular}

TABLE 4

$C P U$ time and iterations (within parenthesis) of BlockIP (using $D^{-1}$ and $\Theta_{0}^{-1}$ preconditioners) and CPLEX available LP methods (Primal Simplex, Dual Simplex, Barrier Method) when solving MNFPNCs. All the nodes have an outflow capacity, i.e., $l=n$.

\begin{tabular}{rrrrrrrrr}
\hline \multirow{2}{*}{$n$} & \multirow{2}{*}{$k$} & \multirow{2}{*}{ n. var. } & \multirow{2}{*}{ n. con. } & \multicolumn{3}{c}{ CPLEX } & \multicolumn{2}{c}{ BlockIP } \\
\cline { 5 - 7 } \cline { 5 - 7 } & & & & Primal Simplex & Dual Simplex & Barrier & $\Theta_{0}^{-1}$ & $D^{-1}$ \\
\hline 150 & 200 & 880030 & 29950 & $75.1(967177)$ & $6.7(86446)$ & $11.8(17)$ & $5.9(29)$ & $5.8(29)$ \\
150 & 400 & 1803230 & 59750 & $257.0(607837)$ & $18.6(163574)$ & $23.8(17)$ & $14.0(32)$ & $12.9(31)$ \\
150 & 600 & 2601030 & 89550 & $426.9(729617)$ & $32.3(249227)$ & $33.6(17)$ & $21.9(34)$ & $20.0(33)$ \\
150 & 800 & 3850430 & 119350 & $585.4(476154)$ & $52.8(330616)$ & $52.0(19)$ & $29.9(33)$ & $27.9(32)$ \\
\hline 300 & 200 & 3587460 & 60100 & $682.9(4310028)$ & $28.3(203780)$ & $85.4(16)$ & $54.9(33)$ & $30.4(34)$ \\
300 & 400 & 7022460 & 119900 & $>3600(8226752)$ & $72.2(413460)$ & $120.3(13)$ & $78.9(37)$ & $66.5(33)$ \\
300 & 600 & 11245260 & 179700 & $>3600(8489927)$ & $118.5(567590)$ & $205.6(15)$ & $140.5(32)$ & $140.1(33)$ \\
300 & 800 & 14862460 & 239500 & $>3600(8408703)$ & $181.6(779265)$ & $284.7(16)$ & $141.2(32)$ & $140.8(40)$ \\
\hline
\end{tabular}

factorization.

4.2. Multicommodity network flow problem with nodal capacities and equal flows. Let $\mathcal{G}=(\mathcal{V}, \mathcal{A})$ be a directed graph, where $\mathcal{V}$ is a set of $n$ vertices or nodes and $\mathcal{A}$ is a set of $n^{\prime}$ arcs, and let $\mathcal{K}$ be a set of $k$ commodities. The multicommodity network flow problem with nodal capacities (MNFPNC from now on) looks for the minimum cost routing of the flows for all the commodities from some source to some destination nodes, imposing capacities on the total outflow at some nodes $h \in \mathcal{C} \subseteq \mathcal{V}$. This problem differs from the standard multicommodity flow problem in that capacities are imposed at nodes, instead of at arcs. The node capacity constraints are

$$
\sum_{i \in \mathcal{K}} \sum_{j \in \mathcal{V}} x_{h j}^{i} \leq b_{h}^{0}, \quad h \in \mathcal{C},
$$

where $b_{h}^{0}$ denotes the capacity of node $h \in \mathcal{C}$. MNFPNC matches the standard formulation (2.1) of primal block-angular problems, by considering $m_{i}=n, n_{i}=n^{\prime}, N_{i}=N \in \mathbb{R}^{(n-1) \times n^{\prime}}$ for all $i=1, \ldots, k$, where $N$ is the node-arc incidence matrix associated to $\mathcal{G}$; and $l=|\mathcal{C}|, L_{i}=L \in \mathbb{R}^{l \times n^{\prime}}$ for all $i=1, \ldots, k$, are derived from $N$ by considering only positive coefficients to obtain (4.2).

Tables 3 and 4 report two computational experiments associated to MNFPNCs of different sizes. The eight instances of Table 3 correspond to problems where $l=0.2 n$ (i.e., only $20 \%$ of nodes are constrained to have an outflow capacity), whereas the eight instances of Table 4 correspond to problems where $l=n$ (all the nodes have capacities). For each instance, the tables provide the number of nodes $n$, number of commodities $k$, the total number of variables ("n.var"), total number of constraints ("n.con."), and the CPU time and (within parentheses) total number of iterations for all the solvers considered: primal simplex, dual simplex and barrier for CPLEX; and BlockIP with both $\Theta_{0}^{-1}$ and $D^{-1}$ preconditioners. In these runs BlockIP computed Newton directions, which is its default option when PCG is used. A time limit of 3600 seconds was considered.

It can be seen from Tables 3 and 4 that the CPU time associated to the primal simplex is always far greater than the ones of the other solvers. The dual simplex is quite competitive and in some instances outperforms CPLEX barrier method and BlockIP. BlockIP with both preconditioners is in general more efficient than CPLEX barrier, although it requires more IPM iterations because it computes Newton directions instead of second or higher order ones. The network size $n$ does not seem to have a substantial effect on the comparative efficiency of the five solvers. Instead the increase in the number of linking constraints (either $l=0.2 n$, in Table 3 or $l=n$, in Table 4) almost doubles the CPU time of the CPLEX barrier method, whereas only slightly affects the specialized IPM. 
TABLE 5

CPU time and iterations (within parenthesis) of BlockIP (using $D^{-1}$ and $\Theta_{0}^{-1}$ preconditioner) and CPLEX available LP methods (Primal Simplex, Dual Simplex, Barrier Method) when solving MEFPNCs. Only $20 \%$ of nodes are constrained to have an outflow capacity, i.e., $l=0.2 n$.

\begin{tabular}{|c|c|c|c|c|c|c|c|c|}
\hline \multirow{2}{*}{$n$} & \multirow{2}{*}{$k$} & \multirow{2}{*}{ n. var. } & \multirow{2}{*}{ n. con. } & \multicolumn{3}{|c|}{ CPLEX } & \multicolumn{2}{|c|}{ BlockIP } \\
\hline & & & & Primal Simplex & Dual Simplex & Barrier & $\Theta_{0}^{-1}$ & $D^{-1}$ \\
\hline 150 & 200 & 880030 & 56830 & $59.3(989973)$ & $5.9(90369)$ & $7.7(16)$ & $6.5(30)$ & $6.8(32)$ \\
\hline 150 & 400 & 1803230 & 113630 & $239.0(516211)$ & $19.5(222725)$ & $17.6(18$ & $11.5(32)$ & $11.5(33)$ \\
\hline 150 & 600 & 2601030 & 170430 & $514.1(563073)$ & $33.9(280339)$ & $42.2(27)$ & $25.4(34)$ & $24.0(33)$ \\
\hline 150 & 800 & 3850430 & 227230 & $635.4(650626)$ & $52.0(406268)$ & $54.1(26)$ & $30.9(32)$ & $30.5(32)$ \\
\hline 300 & 200 & 3587460 & 113860 & $1357.0(958439)$ & $37.9(232976)$ & $44.9(20)$ & $39.6(37)$ & $34.8(34)$ \\
\hline 300 & 400 & & & $>3600.0(132$ & $85.3(45$ & $118.2(29)$ & $70.7(32)$ & $70.1(32)$ \\
\hline 300 & 600 & 11245260 & 341460 & $>3600.0(1213005)$ & $142.5(665822)$ & $155.7(25)$ & $109.2(33)$ & $108.7(34)$ \\
\hline 300 & 800 & 14862460 & 455260 & $>3600.0(1179502)$ & $204.8(860729)$ & $239.8(30)$ & $152.8(34)$ & $150.8(34)$ \\
\hline
\end{tabular}

TABLe 6

$C P U$ time and iterations (within parenthesis) of BlockIP (using $D^{-1}$ and $\Theta_{0}^{-1}$ preconditioner) and CPLEX available LP methods (Primal Simplex, Dual Simplex, Barrier Method) when solving MEFPNCs. All the nodes have an outflow capacity, i.e., $l=n$.

\begin{tabular}{|c|c|c|c|c|c|c|c|c|}
\hline \multirow{2}{*}{$n$} & \multirow{2}{*}{$k$} & \multirow{2}{*}{ n. var. } & \multirow{2}{*}{ n. con. } & \multicolumn{3}{|c|}{ CPLEX } & \multicolumn{2}{|c|}{ BlockIP } \\
\hline & & & & Primal Simplex & Dual Simplex & Barrier & $\Theta_{0}^{-1}$ & $D^{-1}$ \\
\hline 150 & 200 & 880030 & 56950 & $79.0(2320414)$ & $7.5(86446)$ & $11.3(16)$ & $5.9(32)$ & $5.0(32)$ \\
\hline 150 & 400 & 1803230 & 113750 & $296.9(964870)$ & $26.7(163574)$ & $26.7(18)$ & $15.1(33)$ & $14.6(33)$ \\
\hline 150 & 600 & 2601030 & 170550 & $619.9(1839715)$ & $43.0(230553)$ & $96.7(27)$ & $29.6(34)$ & $26.5(33)$ \\
\hline 150 & 800 & 3850430 & 227350 & 891.7 (1061339) & $74.7(249227)$ & $68.0(22)$ & $35.8(33)$ & $34.5(32)$ \\
\hline 300 & 200 & 3587460 & 114100 & $820.8(1184579)$ & $36.9(330616)$ & $87.6(18)$ & $51.1(36)$ & $43.7(34)$ \\
\hline 300 & 400 & 7022460 & 227900 & $>3600.0(4906436)$ & $88.6(203780)$ & $232.8(22)$ & $98.8(34)$ & $80.2(34)$ \\
\hline 300 & 600 & 11245260 & 341700 & $>3600.0(4701108)$ & $177.3(413460)$ & $288.5(21)$ & $125.5(35)$ & $119.0(36)$ \\
\hline 300 & 800 & 14862460 & 455500 & $>3600.0(4904433)$ & 364.9 (1278588) & $480.1(25)$ & $240.0(35)$ & $206.6(34)$ \\
\hline
\end{tabular}

Let us consider a slight modification of the MNFPNCs, obtained by introducing to each commodity a set of equal flow constraints, i.e. constraints requiring that each arc in a specified set $\mathcal{R}_{r}$ must carry the same amount of flow, for every group of arcs $r \in R$; that is, $x_{a_{j}}^{i}=x_{a_{h}}^{i}$, for $i=1 \ldots k$, $a_{j}, a_{h} \in \mathcal{R}_{r}, r=1 \ldots R$ (where $a_{j}, a_{h}$ denote two particular arcs in the network). These constraints arose while modeling some real-life problems, such as water resource system management [22]. We call this problem multicommodity equal flow problem with nodal capacities (MEFPNC from now on). Here we are considering MEFPNCs of different sizes with $R=\mathrm{EF} \cdot n$ groups of arcs having the same flows per each commodity and $\left|\mathcal{R}_{r}\right|=\mathrm{EF} \cdot 100$ number of arcs in each group $r=1 \ldots R$, where EF is a given parameter to control the number of equal flow constraints.

Tables 5 and 6 report two computational experiments associated to these MEFPNCs. As before, the eight instances of Table 5 correspond to problems with $l=0.2 n$, whereas $l=n$ in Table 6 . The parameter EF was set to 0.1 in these instances. The meaning of the columns is the same as in Tables 3 and 4. It is worth mentioning that, for every set $\mathcal{R}_{r}=\left\{a_{1}, a_{2}, \ldots, a_{\left|\mathcal{R}_{r}\right|}\right\}$, and $i \in \mathcal{K}$, the equal flow constraints are formulated as $x_{a_{j}}^{i}=x_{a_{j+1}}^{i}, j=1, \ldots,\left|\mathcal{R}_{r}\right|-1$, instead of $x_{a_{1}}^{i}=x_{a_{j}}^{i}, j=2, \ldots,\left|\mathcal{R}_{r}\right|$. With this formulation we avoid a "dense column" for variables $x_{a_{1}}^{i}$, which makes BlockIP more efficient. CPLEX barrier is not affected by this different reformulation due to its presolving capabilities. The principal angles between linking and block constraints are the same for both formulations, since they span the same subspace (it is easy to show that given the constraints matrices for the two formulations, one can be obtained from the other by simple linear manipulations).

The inclusion of equal flow constraints negatively affects the computational performance of all the considered solvers, though in different proportions. The dual simplex and barrier almost double its CPU times in the largest instances with respect to the MNFPNCs, whereas the ones associated to the specialized IPM slightly increase. In fact, the specialized IPM becomes the most efficient algorithm for this class of problems from $k=400$. Also for the MEFPNCs, as it was for the MNFPNCs, the network size $n$ does not seem to have a substantial effect on the ranking of the five solvers.

As for the two preconditioners of BlockIP, results with $\Theta_{0}^{-1}$ and $D^{-1}$ are very similar in solving MEFPNCs with $l=0.2 n$, as shown in Table 5 . The results with $D^{-1}$ indicate it to be a slightly better preconditioner when $l=n$. In both cases BlockIP outperforms the CPLEX available LP methods. It is worth noting that BlockIP uses an out-of-date sparse linear algebra package for the Cholesky factorization [24], while CPLEX implements highly tuned state-of-the-art factorization routines. Therefore, the performance of BlockIP could be significantly improved by the use of a more 
TABLE 7

Average principal angles between the subspaces generated by the columns of $L^{\top}$ and $N^{\top}$, for each instance of multi-commodity network flow problems with nodal capacities in Tables 3, 4, 5 and 6.

\begin{tabular}{lrrrr}
\hline \multirow{2}{*}{$n$} & \multicolumn{4}{c}{ average principal angles } \\
\cline { 2 - 5 } & Table 3 & Table 4 & Table 5 & Table 6 \\
\hline 150 & 0.8774 & 0.8319 & 0.7958 & 0.8225 \\
300 & 0.8544 & 0.8297 & 0.7732 & 0.8247 \\
\hline
\end{tabular}

TABLE 8

Dimensions and properties of MEFPNCs with small and dense networks.

\begin{tabular}{|c|c|c|c|c|c|c|c|c|}
\hline \# & $n$ & $k$ & $l$ & density & $\mathrm{NC}$ & $\mathrm{EF}$ & n. var. & n. con. \\
\hline 1 & 400 & 2000 & 40 & 0.2 & 0.1 & 0.00 & 32894040 & 798040 \\
\hline 2 & 400 & 2000 & 40 & 0.2 & 0.1 & 0.05 & 32894040 & 998040 \\
\hline 3 & 400 & 2000 & 40 & 0.2 & 0.1 & 0.10 & 32894040 & 1598040 \\
\hline 4 & 400 & 2000 & 40 & 0.2 & 0.1 & 0.15 & 32894040 & 2598040 \\
\hline 5 & 400 & 2000 & 40 & 0.2 & 0.1 & 0.20 & 32894040 & 3998040 \\
\hline 6 & 400 & 2000 & 80 & 0.2 & 0.2 & 0.00 & 32894080 & 798080 \\
\hline 7 & 400 & 2000 & 80 & 0.2 & 0.2 & 0.05 & 32894080 & 998080 \\
\hline 8 & 400 & 2000 & 80 & 0.2 & 0.2 & 0.10 & 32894080 & 1598080 \\
\hline 9 & 400 & 2000 & 80 & 0.2 & 0.2 & 0.15 & 32894080 & 2598080 \\
\hline 10 & 400 & 2000 & 80 & 0.2 & 0.2 & 0.20 & 32894080 & 3998080 \\
\hline 11 & 400 & 2000 & 120 & 0.2 & 0.3 & 0.00 & 32894120 & 798120 \\
\hline 12 & 400 & 2000 & 120 & 0.2 & 0.3 & 0.05 & 32894120 & 998120 \\
\hline 13 & 400 & 2000 & 120 & 0.2 & 0.3 & 0.10 & 32894120 & 1598120 \\
\hline 14 & 400 & 2000 & 120 & 0.2 & 0.3 & 0.15 & 32894120 & 2598120 \\
\hline 15 & 400 & 2000 & 120 & 0.2 & 0.3 & 0.20 & 32894120 & 3998120 \\
\hline 16 & 400 & 2000 & 40 & 0.4 & 0.1 & 0.00 & 64142040 & 798040 \\
\hline 17 & 400 & 2000 & 40 & 0.4 & 0.1 & 0.05 & 64142040 & 998040 \\
\hline 18 & 400 & 2000 & 40 & 0.4 & 0.1 & 0.10 & 64142040 & 1598040 \\
\hline 19 & 400 & 2000 & 40 & 0.4 & 0.1 & 0.15 & 64142040 & 2598040 \\
\hline 20 & 400 & 2000 & 40 & 0.4 & 0.1 & 0.20 & 64142040 & 3998040 \\
\hline 21 & 400 & 2000 & 80 & 0.4 & 0.2 & 0.00 & 64142080 & 798080 \\
\hline 22 & 400 & 2000 & 80 & 0.4 & 0.2 & 0.05 & 64142080 & 998080 \\
\hline 23 & 400 & 2000 & 80 & 0.4 & 0.2 & 0.10 & 64142080 & 1598080 \\
\hline 24 & 400 & 2000 & 80 & 0.4 & 0.2 & 0.15 & 64142080 & 2598080 \\
\hline 25 & 400 & 2000 & 80 & 0.4 & 0.2 & 0.20 & 64142080 & 3998080 \\
\hline 26 & 400 & 2000 & 120 & 0.4 & 0.3 & 0.00 & 64142120 & 798120 \\
\hline 27 & 400 & 2000 & 120 & 0.4 & 0.3 & 0.05 & 64142120 & 998120 \\
\hline 28 & 400 & 2000 & 120 & 0.4 & 0.3 & 0.10 & 64142120 & 1598120 \\
\hline 29 & 400 & 2000 & 120 & 0.4 & 0.3 & 0.15 & 64142120 & 2598120 \\
\hline 30 & 400 & 2000 & 120 & 0.4 & 0.3 & 0.20 & 64142120 & 3998120 \\
\hline
\end{tabular}

recent Cholesky solver.

The information of the average principal angles between the subspaces generated by the columns of $L^{\top}$ and $N^{\top}$ of the instances of Tables 3-6 is reported in Table 7, differentiating for the 150 and 300 nodes instances. These true principal angles were computed with the exact algorithm of Figure 7 in the Appendix, so they are not estimations given by the two methods described in Subsection 3.2. The first observation we deduce from data collected in Table 7 is that the average principal angles are generally stable with respect to the number of nodes. We also see that average principal angles are far from 0 , which may explain why preconditioner $D^{-1}$ outperforms $\Theta_{0}^{-1}$, in general, in Tables 3-6.

We finally generated and solved a set of large instances, considering only the CPLEX barrier solver, and BlockIP with the two preconditioners $\Theta_{0}^{-1}$ and $D^{-1}$, and a new variant (named "automatic" in the tables of results) that selected the best preconditioner according to (3.29), possibly switching to the other preconditioner using the criteria in (3.30). No time limit was set in these runs. The corresponding results are reported in Tables 8-9 and Tables 10-12, for small dense and big sparse networks, respectively. Tables 8 and 10 show the dimensions and characteristics of the instances: columns $l$ provide the number of linking constraints; columns "density" provide the fraction "number of $\operatorname{arcs} /$ maximum number of $\operatorname{arcs}(n(n-1) / 2)$ "; columns "NC" show the fraction of nodal capacity constraints, i.e., "number of node capacities $/ n$ "; and columns "EF" provide the parameter considered for the equal flow constraints. The rest of columns have the same meaning as in previous tables.

The instances of Table 8 with small dense networks were solved with CPLEX, and BlockIP using the Newton direction; Table 9 shows these results. For the instances of Table 10 with large sparse networks, we additionally used BlockIP with the predictor-corrector direction (as discussed below, this was instrumental for the efficiency of BlockIP). These results are reported in Tables 11- 
TABLE 9

Results with BlockIP (using Newton direction for variants $D^{-1}, \Theta_{0}^{-1}$ and automatic selection of preconditioners) and CPLEX barrier for MEFPNCs with small and dense networks.

\begin{tabular}{|c|c|c|c|c|c|c|c|c|c|c|c|c|c|}
\hline \multirow[b]{3}{*}{$\#$} & \multirow{2}{*}{\multicolumn{2}{|c|}{$\frac{\text { CPLEX }}{\text { Barrier }}$}} & \multicolumn{11}{|c|}{ BlockIP Newton direction } \\
\hline & & & \multicolumn{3}{|c|}{$\Theta_{0}^{-1}$} & \multicolumn{3}{|c|}{$D^{-1}$} & \multicolumn{5}{|c|}{ Automatic } \\
\hline & it. & $\mathrm{CPU}$ & it. & PCG & $\mathrm{CPU}$ & it. & PCG & $\mathrm{CPU}$ & it. & $\mathrm{PCG}$ & $\mathrm{CPU}$ & Prec. & $\gamma$ \\
\hline 1 & 17 & 428.32 & 32 & 57 & 424.55 & 33 & 51 & 432.94 & 33 & 51 & 441.49 & $D^{-1}$ & 0.773 \\
\hline 2 & 19 & 461.37 & 35 & 75 & 487.77 & 34 & 55 & 508.19 & 34 & 55 & 455.40 & $D^{-1}$ & 0.771 \\
\hline 3 & 31 & 761.24 & 35 & 88 & 544.80 & 35 & 61 & 538.53 & 35 & 61 & 559.44 & $D^{-1}$ & 0.767 \\
\hline 4 & 30 & 724.38 & 37 & 155 & 706.19 & 37 & 99 & 636.19 & 36 & 103 & 596.13 & $D^{-1}(4)$ & 0.756 \\
\hline 5 & 27 & 708.23 & 37 & 127 & 697.22 & 40 & 90 & 698.52 & 37 & 127 & 721.17 & $\Theta_{0}^{-1}$ & 0.740 \\
\hline 6 & 18 & 402.91 & 33 & 60 & 441.18 & 33 & 50 & 435.00 & 33 & 50 & 432.44 & $D^{-1}$ & 0.803 \\
\hline 7 & 19 & 465.19 & 35 & 82 & 487.26 & 34 & 55 & 456.62 & 34 & 55 & 460.36 & $D^{-1}$ & 0.802 \\
\hline 8 & 29 & 705.41 & 35 & 88 & 542.94 & 36 & 61 & 556.18 & 36 & 61 & 540.66 & $D^{-1}$ & 0.797 \\
\hline 9 & 26 & 704.96 & 38 & 169 & 674.00 & 39 & 106 & 641.39 & 37 & 121 & 639.92 & $D^{-1}(4)$ & 0.788 \\
\hline 10 & 41 & 1071.90 & 38 & 187 & 754.78 & 40 & 119 & 727.62 & 38 & 137 & 717.11 & $D^{-1}(4)$ & 0.774 \\
\hline 11 & 17 & 422.87 & 33 & 62 & 445.08 & 33 & 49 & 440.76 & 33 & 49 & 452.55 & $D^{-1}$ & 0.838 \\
\hline 12 & 20 & 535.59 & 35 & 80 & 491.03 & 34 & 55 & 459.19 & 34 & 55 & 488.47 & $D^{-1}$ & 0.837 \\
\hline 13 & 37 & 961.37 & 36 & 90 & 575.95 & 37 & 59 & 574.79 & 37 & 59 & 591.68 & $D^{-1}$ & 0.833 \\
\hline 14 & 28 & 831.13 & 38 & 180 & 687.14 & 39 & 106 & 651.40 & 39 & 106 & 671.72 & $D^{-1}$ & 0.825 \\
\hline 15 & 32 & 1319.40 & 39 & 199 & 789.96 & 40 & 115 & 731.61 & 40 & 115 & 728.53 & $D^{-1}$ & 0.814 \\
\hline 16 & 28 & 901.33 & 33 & 64 & 678.91 & 33 & 53 & 661.25 & 33 & 53 & 663.92 & $D^{-1}$ & 0.768 \\
\hline 17 & 38 & 1315.00 & 34 & 81 & 712.32 & 34 & 61 & 688.42 & 34 & 61 & 689.50 & $D^{-1}$ & 0.767 \\
\hline 18 & 36 & 1385.90 & 35 & 85 & 752.29 & 35 & 66 & 731.96 & 35 & 66 & 743.15 & $D^{-1}$ & 0.765 \\
\hline 19 & 45 & 1697.40 & 36 & 90 & 818.97 & 36 & 69 & 826.87 & 36 & 69 & 807.50 & $D^{-1}$ & 0.760 \\
\hline 20 & 43 & 1843.00 & 37 & 100 & 939.06 & 37 & 86 & 920.71 & 37 & 86 & 886.39 & $D^{-1}$ & 0.753 \\
\hline 21 & 26 & 949.55 & 32 & 64 & 660.20 & 33 & 54 & 667.20 & 33 & 54 & 667.95 & $D^{-1}$ & 0.794 \\
\hline 22 & 30 & 1210.40 & 34 & 84 & 715.17 & 34 & 62 & 734.03 & 34 & 62 & 692.10 & $D^{-1}$ & 0.793 \\
\hline 23 & 50 & 2124.00 & 35 & 91 & 775.08 & 35 & 66 & 752.99 & 35 & 66 & 741.86 & $D^{-1}$ & 0.791 \\
\hline 24 & 36 & 1461.50 & 36 & 96 & 838.86 & 36 & 70 & 840.70 & 36 & 70 & 798.98 & $D^{-1}$ & 0.787 \\
\hline 25 & 35 & 1606.60 & 38 & 112 & 993.90 & 38 & 83 & 912.51 & 38 & 83 & 907.41 & $D^{-1}$ & 0.782 \\
\hline 26 & 38 & 1349.50 & 33 & 78 & 709.65 & 32 & 54 & 647.39 & 32 & 54 & 652.25 & $D^{-1}$ & 0.835 \\
\hline 27 & 28 & 1328.00 & 34 & 86 & 719.12 & 34 & 62 & 694.62 & 34 & 62 & 694.77 & $D^{-1}$ & 0.834 \\
\hline 28 & 41 & 1656.70 & 36 & 105 & 804.65 & 36 & 69 & 770.40 & 36 & 69 & 768.26 & $D^{-1}$ & 0.832 \\
\hline 29 & 41 & 1757.90 & 37 & 109 & 872.03 & 36 & 70 & 803.45 & 36 & 70 & 803.71 & $D^{-1}$ & 0.828 \\
\hline 30 & 30 & 1564.20 & 38 & 123 & 964.46 & 38 & 81 & 914.56 & 38 & 81 & 916.24 & $D^{-1}$ & 0.823 \\
\hline
\end{tabular}

TABLE 10

Dimensions and properties of MEFPNCs with large and sparse networks.

\begin{tabular}{rrrrrrrrr}
\hline$\#$ & $n$ & $k$ & $l$ & density & NC & EF & n. var. & n. con. \\
\hline 31 & 800 & 2000 & 80 & 0.02 & 0.1 & 0.00 & 13898080 & 1598080 \\
32 & 800 & 2000 & 80 & 0.02 & 0.1 & 0.05 & 13898080 & 1998080 \\
33 & 800 & 2000 & 80 & 0.02 & 0.1 & 0.10 & 13898080 & 3198080 \\
34 & 800 & 2000 & 80 & 0.02 & 0.1 & 0.15 & 13898080 & 5198080 \\
35 & 800 & 2000 & 80 & 0.02 & 0.1 & 0.20 & 13898080 & 7998080 \\
\hline 36 & 800 & 2000 & 160 & 0.02 & 0.2 & 0.00 & 13898160 & 1598160 \\
37 & 800 & 2000 & 160 & 0.02 & 0.2 & 0.05 & 13898160 & 1998160 \\
38 & 800 & 2000 & 160 & 0.02 & 0.2 & 0.10 & 13898160 & 3198160 \\
39 & 800 & 2000 & 160 & 0.02 & 0.2 & 0.15 & 13898160 & 5198160 \\
40 & 800 & 2000 & 160 & 0.02 & 0.2 & 0.20 & 13898160 & 7998160 \\
\hline 41 & 800 & 2000 & 240 & 0.02 & 0.3 & 0.00 & 13898240 & 1598240 \\
42 & 800 & 2000 & 240 & 0.02 & 0.3 & 0.05 & 13898240 & 1998240 \\
43 & 800 & 2000 & 240 & 0.02 & 0.3 & 0.10 & 13898240 & 3198240 \\
44 & 800 & 2000 & 240 & 0.02 & 0.3 & 0.15 & 13898240 & 5198240 \\
45 & 800 & 2000 & 240 & 0.02 & 0.3 & 0.20 & 13898240 & 7998240 \\
\hline 46 & 800 & 2000 & 80 & 0.04 & 0.1 & 0.00 & 26484080 & 1598080 \\
47 & 800 & 2000 & 80 & 0.04 & 0.1 & 0.05 & 26484080 & 1998080 \\
48 & 800 & 2000 & 80 & 0.04 & 0.1 & 0.10 & 26484080 & 3198080 \\
49 & 800 & 2000 & 80 & 0.04 & 0.1 & 0.15 & 26484080 & 5198080 \\
50 & 800 & 2000 & 80 & 0.04 & 0.1 & 0.20 & 26484080 & 7998080 \\
\hline 51 & 800 & 2000 & 160 & 0.04 & 0.2 & 0.00 & 26484160 & 1598160 \\
52 & 800 & 2000 & 160 & 0.04 & 0.2 & 0.05 & 26484160 & 1998160 \\
53 & 800 & 2000 & 160 & 0.04 & 0.2 & 0.10 & 26484160 & 3198160 \\
54 & 800 & 2000 & 160 & 0.04 & 0.2 & 0.15 & 26484160 & 5198160 \\
55 & 800 & 2000 & 160 & 0.04 & 0.2 & 0.20 & 26484160 & 7998160 \\
\hline 56 & 800 & 2000 & 240 & 0.04 & 0.3 & 0.00 & 26484240 & 1598240 \\
57 & 800 & 2000 & 240 & 0.04 & 0.3 & 0.05 & 26484240 & 1998240 \\
58 & 800 & 2000 & 240 & 0.04 & 0.3 & 0.10 & 26484240 & 3198240 \\
59 & 800 & 2000 & 240 & 0.04 & 0.3 & 0.15 & 26484240 & 5198240 \\
60 & 800 & 2000 & 240 & 0.04 & 0.3 & 0.20 & 26484240 & 7998240 \\
\hline & & & & & & & &
\end{tabular}


TABLE 11

Results with BlockIP (using predictor-corrector direction for variants $D^{-1}, \Theta_{0}^{-1}$ and automatic selection of preconditioners) and CPLEX barrier for MEFPNCs with large and sparse networks.

\begin{tabular}{|c|c|c|c|c|c|c|c|c|c|c|c|c|c|}
\hline \multirow[b]{3}{*}{$\#$} & \multirow{2}{*}{\multicolumn{2}{|c|}{$\begin{array}{c}\text { CPLEX } \\
\text { Barrier }\end{array}$}} & \multicolumn{11}{|c|}{ BlockIP predictor-corrector direction } \\
\hline & & & \multicolumn{3}{|c|}{$\Theta_{0}^{-1}$} & \multicolumn{3}{|c|}{$D^{-1}$} & \multicolumn{5}{|c|}{ Automatic } \\
\hline & it. & $\mathrm{CPU}$ & it. & $\mathrm{PCG}$ & $\mathrm{CPU}$ & it. & PCG & $\mathrm{CPU}$ & it. & $\mathrm{PCG}$ & $\mathrm{CPU}$ & Prec. & $\gamma$ \\
\hline 31 & 12 & 495.06 & 22 & 72 & 677.86 & 24 & 79 & 764.06 & 22 & 72 & 691.65 & $\Theta_{0}^{-1}$ & 0.703 \\
\hline 32 & 21 & 796.48 & 24 & 134 & 791.57 & 22 & 90 & 741.25 & 24 & 134 & 814.78 & $\Theta_{0}^{-1}$ & 0.694 \\
\hline 33 & 13 & 613.00 & 23 & 126 & 943.45 & 26 & 197 & 1152.24 & 23 & 126 & 948.45 & $\Theta_{0}^{-1}$ & 0.649 \\
\hline 34 & 32 & 1535.60 & 28 & 149 & 1543.33 & 31 & 307 & 2010.34 & 28 & 149 & 1547.49 & $\Theta_{0}^{-1}$ & 0.589 \\
\hline 35 & 85 & 4442.30 & 21 & 98 & 1472.82 & 192 & 3755 & 18933.60 & 21 & 98 & 1491.84 & $\Theta_{0}^{-1}$ & 0.491 \\
\hline 36 & 12 & 559.93 & 22 & 85 & 696.55 & 23 & 77 & 746.63 & 23 & 77 & 707.57 & $D^{-1}$ & 0.748 \\
\hline 37 & 22 & 1041.20 & 23 & 133 & 774.28 & 26 & 103 & 829.87 & 23 & 133 & 760.36 & $\Theta_{0}^{-1}$ & 0.741 \\
\hline 38 & 22 & 1243.30 & 24 & 166 & 1064.07 & 27 & 220 & 1232.14 & 24 & 166 & 1037.54 & $\Theta_{0}^{-1}$ & 0.707 \\
\hline 39 & 25 & 1611.80 & 23 & 126 & 1310.97 & 39 & 360 & 2385.75 & 23 & 126 & 1282.90 & $\Theta_{0}^{-1}$ & 0.647 \\
\hline 40 & 45 & 2901.50 & 21 & 93 & 1504.07 & 31 & 406 & 2611.41 & 21 & 93 & 1453.75 & $\Theta_{0}^{-1}$ & 0.548 \\
\hline 41 & 11 & 674.28 & 22 & 77 & 679.94 & 23 & 77 & 707.79 & 23 & 77 & 704.19 & $\frac{v}{D^{-1}}$ & 0.804 \\
\hline 42 & 23 & 1359.10 & 24 & 140 & 836.78 & 26 & 103 & 822.08 & 26 & 103 & 830.87 & $D^{-1}$ & 0.798 \\
\hline 43 & 14 & 1050.30 & 24 & 174 & 1038.12 & 30 & 232 & 1312.26 & 30 & 232 & 1322.88 & $D^{-1}$ & 0.768 \\
\hline 44 & 22 & 1597.40 & 22 & 141 & 1253.54 & 58 & 860 & 4049.23 & 22 & 141 & 1270.42 & $\Theta_{0}^{-1}$ & 0.708 \\
\hline 45 & 85 & 5944.70 & 21 & 100 & 1463.74 & 24 & 410 & 2167.35 & 21 & 100 & 1483.09 & $\Theta_{0}^{-1}$ & 0.606 \\
\hline 46 & 13 & 789.73 & 22 & 79 & 1031.86 & 24 & 66 & 1100.24 & 22 & 79 & 1046.23 & $\Theta_{0}^{-1}$ & 0.738 \\
\hline 47 & 29 & 1708.00 & 23 & 90 & 1129.02 & 26 & 75 & 1257.89 & 23 & 90 & 1131.68 & $\Theta_{0}^{-1}$ & 0.733 \\
\hline 48 & 32 & 1985.10 & 24 & 124 & 1348.27 & 28 & 122 & 1577.65 & 24 & 124 & 1352.66 & $\Theta_{0}^{-1}$ & 0.714 \\
\hline 49 & 15 & 1108.80 & 23 & 154 & 1617.12 & 25 & 160 & 1804.16 & 23 & 154 & 1632.36 & $\Theta_{0}^{-1}$ & 0.689 \\
\hline 50 & 15 & 1230.90 & 21 & 124 & 1773.00 & 29 & 250 & 2626.98 & 21 & 124 & 1743.29 & $\Theta_{0}^{-1}$ & 0.652 \\
\hline 51 & 12 & 875.53 & 23 & 77 & 1087.61 & 25 & 68 & 1141.81 & 25 & 68 & 1177.41 & $D^{-1}$ & 0.767 \\
\hline 52 & 26 & 2172.00 & 24 & 104 & 1260.91 & 26 & 74 & 1244.23 & 26 & 74 & 1270.97 & $D^{-1}$ & 0.763 \\
\hline 53 & 29 & 2191.00 & 24 & 131 & 1385.90 & 25 & 99 & 1361.02 & 25 & 99 & 1365.77 & $D^{-1}$ & 0.749 \\
\hline 54 & 24 & 2146.90 & 24 & 204 & 1807.09 & 25 & 199 & 1793.25 & 24 & 204 & 1751.85 & $\Theta_{0}^{-1}$ & 0.723 \\
\hline 55 & 18 & 1733.10 & 23 & 151 & 2020.30 & 26 & 249 & 2309.60 & 23 & 151 & 2075.58 & $\Theta_{0}^{-1}$ & 0.687 \\
\hline 56 & 13 & 1134.40 & 22 & 82 & 1033.35 & 25 & 70 & 1146.59 & 25 & 70 & 1204.39 & $D^{-1}$ & 0.805 \\
\hline 57 & 32 & 2622.70 & 24 & 119 & 1212.28 & 26 & 84 & 1257.95 & 26 & 84 & 1263.93 & $D^{-1}$ & 0.802 \\
\hline 58 & 29 & 2505.10 & 25 & 136 & 1413.30 & 25 & 93 & 1422.24 & 25 & 93 & 1372.66 & $D^{-1}$ & 0.789 \\
\hline 59 & 27 & 2610.70 & 25 & 198 & 1891.78 & 25 & 215 & 1911.14 & 25 & 215 & 1821.69 & $D^{-1}$ & 0.767 \\
\hline 60 & 13 & 1547.80 & 25 & 197 & 2202.88 & 28 & 295 & 2693.02 & 25 & 197 & 2164.69 & $\Theta_{0}^{-1}$ & 0.732 \\
\hline
\end{tabular}

12. For each run, these tables provide the number of IPM iterations (columns "it."), CPU times in seconds (columns "CPU"), overall number of PCG iterations (columns "PCG"); and, for the BlockIP "automatic" variant, the initial estimated principal angle using (3.28) (columns " $\gamma$ "), and the initially selected preconditioner according to (3.29) (columns "Prec."). Column "Prec." also reportsin brackets, and in case of a switching between preconditioners - the particular IPM iteration number where the switch was performed.

Among the 30 instances of Table 9, BlockIP with some variant outperformed CPLEX barrier in 27 instances, and CPLEX barrier was only superior in the three smallest ones: $\# 1, \# 6$ and $\# 11$. The "automatic" variant only selected preconditioner $\Theta_{0}^{-1}$ for instance $\# 5 ; D^{-1}$ was used for the remaining ones since their estimated angles were above the threshold value $\tau_{\gamma}=0.95 \pi / 4=0.74613$ for the rule (3.29). The switching between preconditioners only happened in three instances, $\# 4, \# 9$ and $\# 1$, reducing the CPU time compared to using either $\Theta_{0}^{-1}$ or $D^{-1}$ for all the IPM iterations. In the remaining instances the initially selected preconditioner was applied for all the IPM iterations; and, remarkably, the best preconditioner (the one involving less CPU time and/or less number of PCG iterations) was selected in most instances. In these cases the CPU time of the "automatic" variant incurred in some overhead due to the computation of principal angles. (In some instances the CPU time with the "automatic" variant is sligthly less than that obtained with either $\Theta_{0}^{-1}$ or $D^{-1}$; this (in theory impossible) fact was due to inacuracies of the operating system timing routines. This fact was also observed in results of other tables.)

As for the 30 instances of Table 10, the results obtained with BlockIP computing the Newton direction, reported in Table 12, were not fully satisfactory. It can be observed that CPLEX barrier (whose results are reported in Table 11) usually outperformed BlockIP using the Newton direction with either variant. Nevertheless, even in these unfavourable cases, the "automatic" variant usually selected the best option (by a significant margin in some instances, such as in \#35, \#40, \#45, \#50, \#55 and \#60). Results drastically changed when BlockIP computed predictor-director directions: BlockIP was competititive and outperformed CPLEX in some of the most difficult instances. These 
TABLE 12

Results with BlockIP (using Newton direction for variants $D^{-1}, \Theta_{0}^{-1}$ and automatic selection of preconditioners) and CPLEX barrier for MEFPNCs with large and sparse networks.

\begin{tabular}{|c|c|c|c|c|c|c|c|c|c|c|c|}
\hline \multirow[b]{3}{*}{$\#$} & \multicolumn{11}{|c|}{ BlockIP Newton direction } \\
\hline & \multicolumn{3}{|c|}{$\Theta_{0}^{-1}$} & \multicolumn{3}{|c|}{$D^{-1}$} & \multicolumn{5}{|c|}{ Automatic } \\
\hline & it. & $\mathrm{PCG}$ & $\mathrm{CPU}$ & it. & $\overline{\mathrm{PCG}}$ & $\mathrm{CPU}$ & it. & PCG & $\mathrm{CPU}$ & Prec. & $\gamma$ \\
\hline 31 & 36 & 66 & 946.43 & 37 & 64 & 989.44 & 36 & 66 & 948.33 & $\Theta_{0}^{-1}$ & 0.703 \\
\hline 32 & 37 & 121 & 1021.63 & 37 & 83 & 988.42 & 37 & 121 & 1022.78 & $\Theta_{0}^{-1}$ & 0.694 \\
\hline 33 & 45 & 138 & 1558.14 & 86 & 171 & 2880.19 & 45 & 138 & 1627.43 & $\Theta_{0}^{-1}$ & 0.649 \\
\hline 34 & 40 & 115 & 1883.31 & 53 & 169 & 2516.36 & 40 & 115 & 2008.19 & $\Theta_{0}^{-1}$ & 0.589 \\
\hline 35 & 36 & 81 & 2153.31 & 86 & 191 & 5198.91 & 36 & 81 & 2237.70 & $\Theta_{0}^{-1}$ & 0.491 \\
\hline 36 & 39 & 71 & 1043.15 & 39 & 58 & 1014.32 & 39 & 58 & 1051.49 & $D^{-1}$ & 0.748 \\
\hline 37 & 37 & 129 & 1030.03 & 38 & 84 & 1016.23 & 37 & 129 & 1057.02 & $\Theta_{0}^{-1}$ & 0.741 \\
\hline 38 & 39 & 139 & 1388.36 & 91 & 181 & 3075.57 & 39 & 139 & 1390.82 & $\Theta_{0}^{-1}$ & 0.707 \\
\hline 39 & 43 & 110 & 2037.50 & 160 & 327 & 7524.45 & 43 & 110 & 2048.50 & $\Theta_{0}^{-1}$ & 0.647 \\
\hline 40 & 36 & 80 & 2157.00 & 99 & 207 & 6239.70 & 36 & 80 & 2252.81 & $\Theta_{0}^{-1}$ & 0.548 \\
\hline 41 & 37 & 63 & 1015.59 & 42 & 61 & 1166.39 & 42 & 61 & 1095.37 & $D^{-1}$ & 0.804 \\
\hline 42 & 40 & 142 & 1128.31 & 38 & 84 & 1022.93 & 38 & 84 & 1032.06 & $D^{-1}$ & 0.798 \\
\hline 43 & 38 & 142 & 1376.46 & 52 & 157 & 1843.27 & 52 & 157 & 1830.28 & $D^{-1}$ & 0.768 \\
\hline 44 & 42 & 120 & 2141.33 & 97 & 256 & 4618.71 & 42 & 120 & 2011.55 & $\Theta_{0}^{-1}$ & 0.708 \\
\hline 45 & 37 & 79 & 2297.12 & 93 & 209 & 5749.08 & 37 & 79 & 2237.28 & $\Theta_{0}^{-1}$ & 0.606 \\
\hline 46 & 34 & 62 & 1434.37 & 33 & 52 & 1330.78 & 34 & 62 & 1385.14 & $\Theta_{0}^{-1}$ & 0.738 \\
\hline 47 & 35 & 97 & 1546.51 & 36 & 64 & 1554.71 & 35 & 97 & 1535.42 & $\Theta_{0}^{-1}$ & 0.733 \\
\hline 48 & 37 & 140 & 1843.27 & 40 & 97 & 1999.25 & 37 & 140 & 1843.39 & $\Theta_{0}^{-1}$ & 0.714 \\
\hline 49 & 36 & 128 & 2105.54 & 38 & 128 & 2364.30 & 36 & 128 & 2181.78 & $\Theta_{0}^{-1}$ & 0.689 \\
\hline 50 & 36 & 101 & 2527.68 & 55 & 160 & 4030.74 & 36 & 101 & 2517.88 & $\Theta_{0}^{-1}$ & 0.652 \\
\hline 51 & 33 & 65 & 1404.61 & 33 & 51 & 1329.42 & 33 & 51 & 1335.45 & $D^{-1}$ & 0.767 \\
\hline 52 & 36 & 99 & 1545.08 & 37 & 64 & 1565.00 & 37 & 64 & 1568.63 & $D^{-1}$ & 0.763 \\
\hline 53 & 38 & 142 & 1888.47 & 38 & 95 & 1801.29 & 38 & 95 & 1874.87 & $D^{-1}$ & 0.749 \\
\hline 54 & 38 & 174 & 2284.05 & 50 & 172 & 2981.77 & 38 & 174 & 2278.19 & $\Theta_{0}^{-1}$ & 0.723 \\
\hline 55 & 38 & 125 & 2709.45 & 62 & 194 & 4374.40 & 38 & 125 & 2665.91 & $\Theta_{0}^{-1}$ & 0.687 \\
\hline 56 & 34 & 65 & 1378.88 & 35 & 52 & 1424.71 & 35 & 52 & 1399.58 & $D^{-1}$ & 0.805 \\
\hline 57 & 36 & 104 & 1544.71 & 37 & 66 & 1538.87 & 37 & 66 & 1544.03 & $D^{-1}$ & 0.802 \\
\hline 58 & 38 & 144 & 1856.94 & 40 & 90 & 1872.33 & 40 & 90 & 1936.99 & $D^{-1}$ & 0.789 \\
\hline 59 & 38 & 166 & 2265.80 & 49 & 161 & 2836.92 & 49 & 161 & 2942.40 & $D^{-1}$ & 0.767 \\
\hline 60 & 37 & 164 & 2691.99 & 69 & 217 & 4849.53 & 37 & 164 & 2753.61 & $\Theta_{0}^{-1}$ & 0.732 \\
\hline
\end{tabular}

results are shown in Table 11. Although predictor-corrector directions for PCG-based IPMs are not as competitive as for Cholesky-based ones (since we have no factorization to reuse), in these instances the number of PCG iterations was small enough to make it worth solving twice the normal equations for a better direction. As in previous cases, the "automatic" variant selected in almost all cases the best preconditioners (some notable results are observed for instances $\# 34, \# 35$, \#39, \#40, \#44, $\# 45$, \#50). We also observe that the more (equal-flow block) constraints, the better $\Theta_{0}^{-1}$ is. This is consistent with theory: the more constraints in $N_{i}$, the "larger" is the subspace spanned by their columns, and then principal angles between the rows of $L_{i}$ and $N_{i}$ are smaller (i.e., closer to 0), which means that $\Theta_{0}^{-1}$ is closer to be the exact preconditioner. From Table 11, we see that BlockIP outperformed CPLEX in 19 of the 30 instances, and by a significant margin in some cases. These results could be improved if larger instances would have been tested. For instance, in a tough instance (not included in the above tables) of $n=4000, k=2000$, density $=0.001, \mathrm{NC}=0.2$, with no equal flow constraints, CPLEX barrier spent about 37000 seconds (requiring 85 Gigabytes of memory), while BlockIP found a solution in 11000 seconds (using 15 Gigabytes of memory).

It is worth remarking that the density of $D$ may negatively affect the performance of preconditioner $D^{-1}$, possibly involving a costly Cholesky factorization, whereas $\Theta_{0}$ is always diagonal. However, in all the MNFPNCs and MEFPNCs instances tested in this work, $D$ is also diagonal. Therefore the different performance of preconditioners is mainly explained by the geometrical relations between the subspaces spanned by the linking and block constraints (and their principal angles). Yet, the computation of $\Theta_{0}$ will always be less costly than that of $D$ : even in the best case for $D$ (that is, when $L_{i}=I$ ), we have $D=\Theta_{0}+\sum_{i=1}^{k} \Theta_{i}$.

5. Conclusions. This work showed that the angles between the subspaces generated by the diagonal blocks and the linking constraints may explain the effectiveness of two complementary preconditioners, namely $\Theta_{0}^{-1}$ and $D^{-1}$, in the specialized IPM for block-angular problems. It was also shown that the evolution of principal angles along the IPM iterations rely on the diagonal $\Theta$ matrix. Two methods for the estimation of principal angles were developed and used to predict ex-ante which 


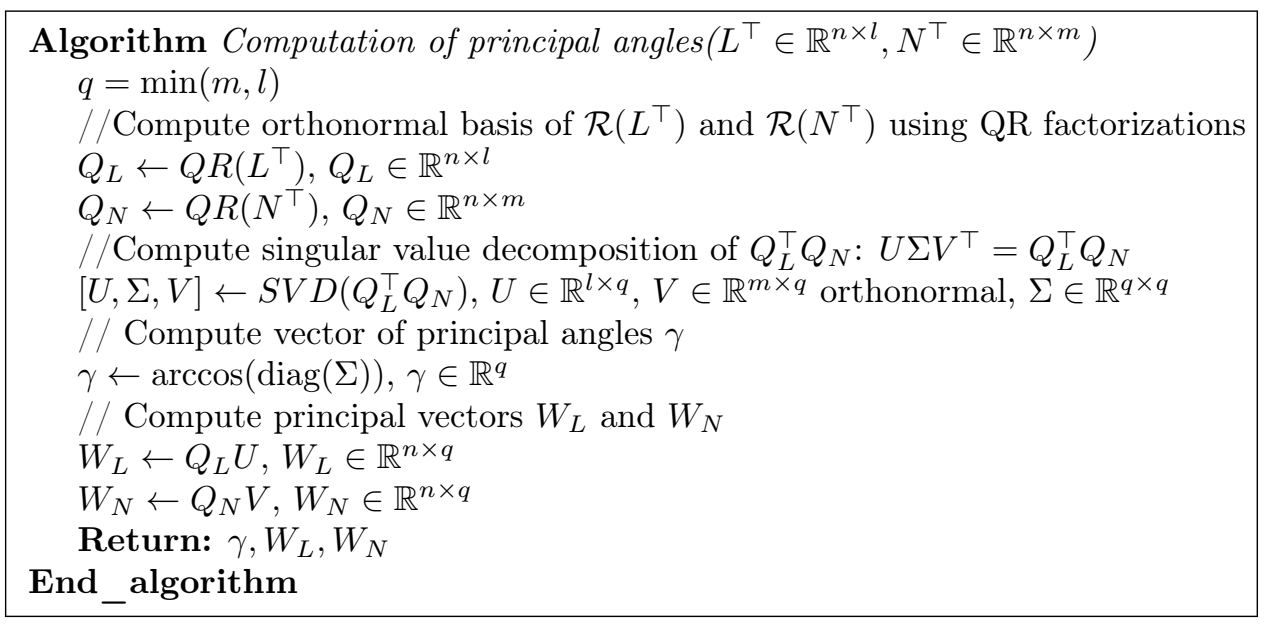

FIG. 7. Algorithm to compute principal angles between column spaces of $L^{\top}$ and $N^{\top}$.

preconditioner to be used for each particular instance. Algebraical properties of the two complementary preconditioners were supported by numerical results. We also analyzed the performance of the specialized IPM with the two preconditioners in the solution of the multicommodity network flow problem with nodal capacities and equal flows, observing that it outperforms all of the available CPLEX methods in some of the largest instances.

$\Theta_{0}^{-1}$ and $D^{-1}$ have shown to be the exact preconditioners when a linking constraint belongs to respectively the range and null space of the matrix of block constraints. Since any vector defining a linking constraint can be decomposed as the sum of two orthogonal vectors belonging to the range and null space of the matrix of block constraints, it might be worth to exploit this fact to see whether a multipreconditioner based on the two above can be obtained, following for instance [8]. This is part of the further research to be done.

Alternative more efficient and accurate methods for the estimation of the principal angles (e.g., considering a sample or representative subset of linking constraints), and applying the specialized IPM (either with $\Theta_{0}^{-1}, D^{-1}$ or combining both) to other problems are also part of the future research.

Acknowledgments. We would like to acknowledge the two anonymous reviewers for his valuable suggestions, that improved the results in the manuscript and fixed several issues.

Appendix. Computation of principal angles.

Principal angles between two subspaces can be computed by the singular value decomposition, as shown by next theorem from [6] (procedure also described in [19, Chapter 12]):

TheOREm A.1. Let the columns of matrices $Q_{L} \in \mathbb{R}^{n \times l}$ and $Q_{N} \in \mathbb{R}^{n \times m}$ form orthonormal bases for the subspaces $\mathcal{L}$ and $\mathcal{N}$, correspondingly. Principal vectors $u \in \mathbb{R}^{n}$ and $v \in \mathbb{R}^{n}$ must verify $u=Q_{L} \widetilde{u}$ and $v=Q_{N} \widetilde{v}$, where $\widetilde{u} \in \mathbb{R}^{l}$ and $\widetilde{v} \in \mathbb{R}^{m}$ are left and right singular vectors of $Q_{L}^{\top} Q_{N}$, associated to the singular value $\cos (\gamma(u, v))$, that is to say, $\left(Q_{L}^{\top} Q_{N}\right) \widetilde{v}=\cos (\gamma(u, v)) \widetilde{u}$.

The algorithm of Figure 7 outlines how to compute the principal angles of column spaces of matrices $L^{\top} \in \mathbb{R}^{n \times l}$ and $N^{\top} \in \mathbb{R}^{n \times m}$. The algorithm is just provided for completeness: as shown in Subsection 3.2, it does not need to be used in practice in our approach. In fact, its use would be prohibitive since the required singular value decomposition is computationally very expensive for large optimization problems.

\section{REFERENCES}

[1] F. Babonneau, O. Du Merle, and J.-P. Vial, Solving large-scale linear multicommodity flow problems with an active set strategy and proximal-ACCPM, Oper. Res., 54 (2006), pp. 184-197.

[2] F. Babonneau And J.-P. Vial, ACCPM with a nonlinear constraint and an active set strategy to solve nonlinear multicommodity flow problems, Math. Prog., 120 (2009), pp. 179-210.

[3] S. Bellavia, J. Gondzio, and B. Morini, B., A matrix-free preconditioner for sparse symmetric positive definite systems and least-squares problems, SIAM J. Sci. Comp., 35 (2013), pp. A192-A211.

[4] L. Bergamaschi, J. Gondzio, J., and G. Zilli, Preconditioning indefinite systems in interior point methods for optimization, Comput. Optim. Appl., 28 (2004), pp. 149-171.

[5] D. Bienstock, Potential Function Methods for Approximately Solving Linear Programming Problems. Theory and Practice, Kluwer, Boston, MA, 2002.

[6] A. Buörck And G.H. Golub, Numerical methods for computing angles between linear subspaces, Math. Comp., 27 (1977), pp. 579-594. 
[7] S. Bocanegra, J. Castro, and A.R.L. Oliveira, Improving an interior-point approach for large block-angular problems by hybrid preconditioners, Eur. J. Oper. Res., 231 (2013), pp. 263-273.

[8] R. Bridson and C. Greif, A multipreconditioned conjugate gradient algorithm, SIAM J Matrix Anal. Appl., 27 (2006), pp. 1056-1068.

[9] Y. CaO, C.D. Laird, and V.M. Zavala, Clustering-based preconditioning for stochastic programs, Comput. Optim. Appl., 64 (2016), pp. 379-406.

[10] J. CAstro, A specialized interior-point algorithm for multicommodity network flows, SIAM J. Optim., 10 (2000), pp. 852-877.

[11] J. CASTRO, An interior-point approach for primal block-angular problems, Comput. Optim. Appl., 36 (2007), pp. $195-219$.

[12] J. CAstro, Interior-point solver for convex separable block-angular problems, Optim. Method. Softw., 31 (2016), pp. 88-109.

[13] J. Castro and J. Cuesta, Quadratic regularizations in an interior-point method for primal block-angular problems, Math. Prog., 130 (2011), pp. 415-445.

[14] J. Castro and N. Nabona, An implementation of linear and nonlinear multicommodity network flows, Eur. J. Oper. Res., 92 (1996), pp. 37-53.

[15] J. Castro And S. Nasini, Mathematical programming approaches for classes of random network problems, Eur. J. Oper. Res., 245 (2015), pp. 402-414.

[16] J. Castro, S. Nasini and F. Saldanha-da-Gama, A cutting-plane approach for large-scale capacitated multiperiod facility location using a specialized interior-point method, Math. Prog., (2016), doi:10.1007/s10107016-1067-6.

[17] A. Frangioni, and G. Gallo, A bundle type dual-ascent approach to linear multicommodity min cost flow problems, INFORMS J. Comput., 11 (1999), pp. 370-393.

[18] A. Frangioni and C. Gentile, New preconditioners for KKT systems of network flow problems, SIAM J. Optim., 14 (2004), pp. 894-913.

[19] G.H. Golub and C.F. Van Loan, Matrix Computations, Third Ed., Johns Hopkins Univ. Press, Baltimore, MD, 1996.

[20] J. Gondzio, Convergence analysis of an inexact feasible interior point method for convex quadratic programming, SIAM J. Optim., 23 (2013), pp. 1510-1527.

[21] J. Gondzio and R. Sarkissian, Parallel interior-point solver for structured linear programs, Math. Prog., 96 (2003), pp. 561-584.

[22] A. Manca, G. Sechi, And P. Zuddas, Water supply network optimisation using equal flow algorithms, Water Resour. Manag., 24 (2010), pp. 3665-3678.

[23] R.D. McBride, Progress made in solving the multicommodity flow problem, SIAM J. Optim., 8 (1998), pp. 947-955.

[24] E. NG, And B.W. Peyton, Block sparse Cholesky algorithms on advanced uniprocessor computers, SIAM J. Sci. Comput., 14 (1993), pp. 1034-1056.

[25] A.R.L. Oliveira, and D.C. Sorensen, A new class of preconditioners for large-scale linear systems from interior point methods for linear programming, Linear Algebra Appl., 394 (2005), pp. 1-24.

[26] J.M. Ortega, Introduction to Parallel and Vector Solutions of Linear Systems, Plenum Press, New York, NY, 1988.

[27] A. Ouorou, A proximal cutting plane method using Chebychev center for nonsmooth convex optimization, Math. Prog., 119 (2009), pp. 239-271.

[28] M.G.C. Resende and G. Veiga, An implementation of the dual affine scaling algorithm for minimum-cost flow on bipartite uncapacitated networks, SIAM J. Optim., 3 (1993), pp. 516-537.

[29] S.J. Wright, Primal-Dual Interior-Point Methods, SIAM, Philadelphia, PA, 1996. 